\title{
Generalized transition fronts for one-dimensional almost periodic Fisher-KPP equations.
}

\author{
Grégoire Nadin * Luca Rossi ${ }^{\dagger}$
}

October 16, 2018

\begin{abstract}
This paper investigates the existence of generalized transition fronts for Fisher-KPP equations in one-dimensional, almost periodic media. Assuming that the linearized elliptic operator near the unstable steady state admits an almost periodic eigenfunction, we show that such fronts exist if and only if their average speed is above an explicit threshold. This hypothesis is satisfied in particular when the reaction term does not depend on $x$ or (in some cases) is small enough. Moreover, except for the threshold case, the fronts we construct and their speeds are almost periodic, in a sense.

When our hypothesis is no longer satisfied, such generalized transition fronts still exist for an interval of average speeds, with explicit bounds.

Our proof relies on the construction of sub and super solutions based on an accurate analysis of the properties of the generalized principal eigenvalues.
\end{abstract}

Key-words: generalized transition fronts, almost periodic, Fisher-KPP equation, generalized principal eigenvalues.

AMS classification: 35B08, 35B15, 35B40, 35B50, 35C07, 35K57, 47E05

The research leading to these results has received funding from the European Research Council under the European Union's Seventh Framework Programme (FP/2007-2013) / ERC Grant Agreement n.321186 - ReaDi -Reaction-Diffusion Equations, Propagation and Modelling.

\section{Introduction and main results}

\section{$1.1 \quad$ Introduction}

We are concerned with one-dimensional Fisher-KPP equations of the type

$$
u_{t}-\left(a(x) u_{x}\right)_{x}=c(x) u(1-u), \quad t \in \mathbb{R}, x \in \mathbb{R},
$$

\footnotetext{
${ }^{*}$ CNRS, UMR 7598, Laboratoire Jacques-Louis Lions, F-75005 Paris, France

${ }^{\dagger}$ Dipartimento di Matematica, Università di Padova, via Trieste 63, 35121 Padova, Italy
} 
with coefficients $a \in \mathcal{C}^{1}(\mathbb{R}), c \in \mathcal{C}(\mathbb{R})$ satisfying the following assumptions:

$$
\inf _{\mathbb{R}} a>0, \quad \inf _{\mathbb{R}} c>0, \quad a, a^{\prime}, c \text { are almost periodic. }
$$

These hypotheses will always be understood throughout the paper without further mention. We use Bochner's definition of almost periodic functions.

Definition 1.1. [13] A function $a: \mathbb{R} \rightarrow \mathbb{R}$ is almost periodic (a.p. in the sequel) if from any sequence $\left(x_{n}\right)_{n \in \mathbb{N}}$ in $\mathbb{R}$ one can extract a subsequence $\left(x_{n_{k}}\right)_{k \in \mathbb{N}}$ such that $a\left(x_{n_{k}}+x\right)$ converges uniformly in $x \in \mathbb{R}$.

A function $U=U(z, x)$ is a.p. in $x$ uniformly with respect to $z \in \mathbb{R}$ if from any sequence $\left(x_{n}\right)_{n \in \mathbb{N}}$ in $\mathbb{R}$ one can extract a subsequence $\left(x_{n_{k}}\right)_{k \in \mathbb{N}}$ such that $U\left(z, x_{n_{k}}+x\right)$ converges uniformly in $(z, x) \in \mathbb{R} \times \mathbb{R}$.

When $a$ and $c$ does not depend on $x$, this equation was investigated in the pioneering papers of Kolmogorov, Petrovsky and Piskunov [19] and Fisher [17], who addressed the issue of the existence of special solutions, called travelling fronts: positive solutions of (11) of the form $u(t, x)=U(x-w t)$ satisfying $U(-\infty)=1$ and $U(+\infty)=0$, with unknowns $U$ (the profile) and $w$ (the speed). Such solutions exist if and only if $w \geq 2 \sqrt{a c}=: w^{*}$. Moreover, the front with minimal speed $w^{*}$ attracts, in a sense, the solutions of the Cauchy problem associated with the initial datum $\mathbb{1}_{(-\infty, 0)}$, see [19]. The existence of travelling fronts was extended to more general types of nonlinearities and multi-dimensional equations by Aronson and Weinberger [2].

When $a$ and $c$ are positive functions of $x$ which are periodic, with the same period, such a heterogeneity should be taken into account in the definition of the front, giving rise to the notion of pulsating travelling front: a positive solution of the form $u(t, x)=U(x-w t, x)$, where $U=U(z, x)$ is periodic in $x, U(-\infty, x)=1$ and $U(+\infty, x)=0$ uniformly in $x$. Analogously to the case of $x$-independent coefficients, pulsating travelling fronts exist if and only if $w \geq w^{*}$, where now $w^{*}>0$ is expressed in terms of the periodic principal eigenvalues of some linear operators, see [3]. This result holds true for more general types of nonlinearities and in multidimensional media [3, 6, 49, as well as when the coefficients are not only periodic in $x$ but also in $t$ [27, 33] .

An increasing attention has been paid to the case of general heterogeneous coefficients in the 2000's. A generalization of the notion of travelling fronts has been given by Berestycki and Hamel [4, 5].

Definition 1.2. 4, 5] A generalized transition front of equation (11) is a time-global solution $u$ for which there exists a function $X: \mathbb{R} \rightarrow \mathbb{R}$ such that

$$
\lim _{x \rightarrow-\infty} u(t, x+X(t))=1, \quad \lim _{x \rightarrow+\infty} u(t, x+X(t))=0, \quad \text { uniformly in } t \in \mathbb{R} .
$$

We say that $u$ has an average speed $w \in \mathbb{R}$ if it holds true that

$$
\lim _{t-s \rightarrow+\infty} \frac{X(t)-X(s)}{t-s}=w .
$$


Travelling fronts and pulsating travelling fronts are particular instances of generalized transition fronts, with $X(t)=w t$.

The existence of generalized transition fronts for 1-dimensional heterogeneous reactiondiffusion equations with ignition-type nonlinearities has been derived in [34, 25], and their stability in [24]. It is also known for bistable time-heterogeneous equations [44]. For FisherKPP equations such as (1), generalized transition waves do not exist in general when the reaction term $c=c(x)$ is heterogeneous [32], but they do if only the diffusion term $a=a(x)$ is heterogeneous [50] or if the coefficients $a=a(t)$ and $c=c(t)$ are time heterogeneous [29] (see also [45] dealing with time uniquely ergodic coefficients). This leads to an alternative notion of critical travelling wave given by the first author in [28] for 1-dimensional equations. The situation is much more complicated in multi-dimensional media. Some conditions have been provided guaranteeing the existence of generalized transition fronts for partially periodic equations of ignition-type [51] or for space-time heterogeneous Fisher-KPP equations under a space periodicity assumption [30, 41, 47]. However, for general heterogeneous ignition-type equations in $\mathbb{R}^{N}, N \geq 3$, generalized transition fronts do not exist in general [52], unlike the 1-dimensional framework.

In order to go further in the investigation of fronts in heterogeneous Fisher-KPP equations, we are thus lead to consider specific classes of heterogeneity since generalized transition fronts do not exist in general for such equations. The aim of the present paper is to understand the case of spatial a.p. coefficients in dimension 1 . The only related results in the literature we are aware of are Shen's [42, 43], concerning bistable equations with a time a.p. reaction term, a typical example being

$$
u_{t}=u_{x x}+u(1-u)(u-\theta(t))
$$

where $\theta$ is an a.p. function such that $0<\theta<1$ in $\mathbb{R}$. This is quite a different problem, but we note that the type of solutions obtained by Shen are indeed similar to the one of Theorem 1.4 below. However, the construction is very different: Shen uses a stability approach, which is well-fitted for bistable equations, combined with a parabolic zero number argument, while in the present paper we use a sub and super solutions method. We also mention here the work of Lou and Chen [23] on curved travelling fronts for the curvature flow equation with space a.p. coefficients, which is known to be an asymptotic limit of bistable equations.

Our approach here is inspired by the case of periodic coefficients (see [27] for example), where the sub and super solutions are constructed starting from exponential solutions of the linearized equation around the unstable steady state $u \equiv 0$. In the periodic case, this naturally leads to consider some eigenvalue problems for linear operators. The main difficulty here is that it is not clear whether an elliptic operator with a.p. coefficients admits classical eigenvalues and, even when it does, there could exist multiple bounded eigenfunctions and they might not be a.p. [40. We will thus make use of particular exponentially decreasing solutions of linear problems (see Proposition 1.3) and of generalized principal eigenvalues (defined by (21)- (22) below). 


\subsection{Statement of the results}

Our characterization of the minimal average speed of generalized transition fronts involves, as in periodic media [6, 27], the eigenfunctions of the linearized operator near the unstable steady state $u \equiv 0$, that is,

$$
\mathcal{L} \phi:=\left(a(x) \phi^{\prime}\right)^{\prime}+c(x) \phi .
$$

Unlike in the periodic case, here we do not dispose of the compactness properties that allow one to define the eigenvalues in a classical sense. In particular, we cannot apply the KreinRutman theorem providing the principal eigenvalue. Thus, we consider the following notion of generalized principal eigenvalue, introduced by Berestycki, Nirenberg and Varadhan in [9]:

$$
\lambda_{1}:=\inf \left\{\lambda \in \mathbb{R}, \exists \phi \in \mathcal{C}^{2}(\mathbb{R}), \phi>0, \mathcal{L} \phi \leq \lambda \phi \text { in } \mathbb{R}\right\}
$$

An equivalent definition was previously given by Agmon [1] for divergence form operators on Riemannian manifolds and, for general operators, by Nussbaum and Pinchover [35, building on a result by Protter and Weinberger [39]. In the sequel, we will also make use of a characterization through a Rayleigh quotient derived in [1], which readily implies that $\lambda_{1}$ is the infimum of the spectrum of $-\mathcal{L}$ on $L^{2}(\mathbb{R})$, and also yields $\lambda_{1} \geq \inf c$ (see Proposition 2.1 below). In particular, under the standing assumptions, there holds

$$
\lambda_{1}>0
$$

Besides the almost periodicity of the coefficients, we will assume the following.

Hypothesis 1. The operator $\mathcal{L}$ admits an a.p. positive eigenfunction, that is, there exists an a.p. positive function $\varphi_{1} \in \mathcal{C}^{2}(\mathbb{R})$ such that $\mathcal{L} \varphi_{1}=\lambda_{1} \varphi_{1}$ in $\mathbb{R}$.

The fact that the eigenvalue associated with an a.p. positive eigenfunction is necessarily the generalized principal eigenvalue $\lambda_{1}$ follows from a Bloch-type property (see [10, Theorem 1.7]). In the periodic case, Hypothesis 1 trivially holds with $\varphi_{1}$ equal to the periodic principal eigenvalue of $\mathcal{L}$. We will see in Section 1.3 that Hypothesis 1 is equivalent to require the existence of a bounded eigenfunction with positive infimum. Let us point out that, for arbitrary operators, there always exists a positive solution of $\mathcal{L} \varphi=\lambda \varphi_{1}$ in $\mathbb{R}$ [1, 10], but it might not be a.p., bounded, nor with a positive infimum.

Checking Hypothesis 1 is actually a difficult task in general. Sorets and Spencer [48] showed that, when $a \equiv 1$ and $c(x)=K(\cos (2 \pi x)+\cos (2 \pi \alpha x))$ with $\alpha \notin \mathbb{Q}$ and $K$ large enough, the Lyapounov exponent of $\phi(n+1)-2 \phi(n)+\phi(n-1)+c(n) \phi(n)=\lambda \phi(n)$ in $\mathbb{Z}$ is strictly positive, for any $\lambda \in \mathbb{R}$. This implies, through Ruelle-Oseledec's theorem, that any solution of this equation should either blow up or decay to zero exponentially, contradicting a possible almost periodicity. This example is especially striking because the above function $c$ is quasiperiodic. It involves the discrete Laplace operator, but we believe that such a phenomenon could also occur for the continuous equation $\mathcal{L} \phi=\lambda_{1} \phi$. Actually, [48] also deals with the continuous Laplace operator, but excludes in this framework some eigenvalues and we were not able to determine if the principal eigenvalue $\lambda_{1}$ is among them.

On the other hand, Hypothesis 1 holds in two relevant cases, where generalized transition fronts were not previously obtained: 
1. $c$ is constant.

2. $a$ and $c$ are quasiperiodic and their periods satisfy the non-degeneracy diophantine condition (9) below.

Indeed, in the first case Hypothesis 11 holds because $\mathcal{L}$ admits constant eigenfunctions. The second case follows from a result by Kozlov [20, that we recall in Section 1.3, The optimality of Hypothesis 1, and of a weaker one based on the theory of critical operators, will be discussed in Section 1.3

We will need the following description of the other eigenlevels.

Proposition 1.3. The following properties hold for all $\gamma>\lambda_{1}$ :

(i) There exists a unique positive solution $\phi_{\gamma} \in \mathcal{C}^{2}(\mathbb{R})$ of

$$
\mathcal{L} \phi_{\gamma}=\gamma \phi_{\gamma} \quad \text { in } \mathbb{R}, \quad \phi_{\gamma}(0)=1, \quad \lim _{x \rightarrow+\infty} \phi_{\gamma}(x)=0 .
$$

(ii) There exists the limit $\mu(\gamma):=-\lim _{x \rightarrow \pm \infty} \frac{1}{x} \ln \phi_{\gamma}(x)>0$.

We are now in position to state our main result.

Theorem 1.4. Let $\mu(\gamma)$ be as in Proposition 1.3. Then, under Hypothesis 1, there exists

$$
w^{*}:=\min _{\gamma>\lambda_{1}} \frac{\gamma}{\mu(\gamma)}>0
$$

and the following properties hold:

(i) For all $w \geq w^{*}$ there exists a time-increasing generalized transition front with average speed $w$; for $w>w^{*}$, the front can be written as $u(t, x)=U\left(\int_{0}^{x} \sigma-t, x\right)$, where $\sigma \in \mathcal{C}(\mathbb{R})$ is a.p. and has average $1 / w$ and $U=U(z, x)$ is a.p. in $x$ uniformly in $z \in \mathbb{R}$.

(ii) There are no generalized transition fronts with average speed $w<w^{*}$.

Roughly speaking, the profile $U$ and the function $\sigma$ expressing the front in the supercritical case $w>w^{*}$ inherit the almost periodicity of the coefficients. Whether or not an analogous property holds true in the case $w=w^{*}$ is left as open question. Moreover, in such case, the front being constructed as a critical travelling wave, in the sense of [28], we conjecture that it is attractive for Heaviside type initial data (see the discussion about this topic in [28]).

We derive the non-existence result as a consequence of an estimate of the propagation of the interface $X(t)$ of generalized transition fronts which is valid even without assuming the existence of an average speed (see Proposition 3.9 below).

Hypothesis 1 allows us to prove the following key property:

$$
\lim _{\gamma \searrow \lambda_{1}} \mu(\gamma)=0
$$


This limit is always well-defined since $\gamma \mapsto \mu(\gamma)$ will be proved to be nondecreasing and nonnegative (see Lemma 2.5). If Hypothesis 1 fails, then exponentially localized eigenfunctions may arise [48, meaning that this limit might be positive.

If we drop Hypothesis 1 we are still able to obtain a partial existence result.

Theorem 1.5. Let $\mu(\gamma)$ be as in Proposition 1.3 and set

$$
w^{*}:=\inf _{\gamma>\lambda_{1}} \frac{\gamma}{\mu(\gamma)}, \quad \underline{w}:=\frac{\lambda_{1}}{\underline{\mu}}, \quad \text { where } \underline{\mu}:=\lim _{\gamma \searrow \lambda_{1}} \mu(\gamma) .
$$

The following properties hold:

(i) If $w^{*}<\underline{w}$ then for all $w \in\left[w^{*}, \underline{w}\right)$, there exists a time-increasing generalized transition front with average speed $w$; for $w>w^{*}$, the front can be written as $u(t, x)=U\left(\int_{0}^{x} \sigma-t, x\right)$, where $\sigma \in \mathcal{C}(\mathbb{R})$ is a.p. and has average $1 / w$ and $U=U(z, x)$ is a.p. in $x$ uniformly in $z \in \mathbb{R}$.

(ii) There are no generalized transition fronts with average speed $w<w^{*}$.

Since Hypothesis 1 entails (6), which in turn yields $\underline{w}=+\infty$, Theorem 1.4 will follow from Theorem 1.5, after showing that $w^{*}$ is attained in that case, see Remark 1. We will also show that (6) holds when $c$ is constant using an alternative direct argument, see Remark 2 ,

The above results leave open several interesting problems:

- Do they extend to multi-dimensional equations? The construction of the $\phi_{\gamma}$ 's in Proposition 1.3 strongly relies on 1D arguments, but are there analogous solutions in higher dimensions?

- Is it possible to construct a rigorous example where $\mu>0$ and, in this case, are there generalized transition fronts with average speed $w>\underline{w}$ ?

- What are the properties of the critical front with speed $w=w^{*}$ ? Does it have a.p. profile? Is it attractive, in a sense, for the Cauchy problem?

We conclude with a small lemma ensuring that the sufficient condition for the existence result in Theorem 1.5(i) is fulfilled up to a constant perturbation of the zero order term.

Lemma 1.6. For $c_{0}$ large enough the quantities $\underline{w}$ and $w^{*}$ provided by Theorem 1.5 for the equation (11) with $c(x)$ replaced by $c(x)+c_{0}$ satisfy $w^{*}<\underline{w}$.

\subsection{Optimality of Hypothesis 1}

In this section we discuss Hypothesis 1 and a generalization. This involves the notion of limit operator associated with $\mathcal{L}$, that is, an operator of the type

$$
\mathcal{L}^{*} \phi:=\left(a^{*}(x) \phi^{\prime}\right)^{\prime}+c^{*}(x) \phi,
$$

where $a^{*}(x)$ and $c^{*}(x)$ are the limits as $n \rightarrow \infty$ of $a\left(x+x_{n}\right)$ and $c\left(x+x_{n}\right)$, for some sequence $\left(x_{n}\right)_{n \in \mathbb{N}}$ in $\mathbb{R}$. We know from [7, Lemma 5.6] that, being $a$ and $c$ a.p., the generalized principal eigenvalue of any limit operator $\mathcal{L}^{*}$ coincides with the one of $\mathcal{L}: \lambda_{1}$. 
Hypothesis 2. For any limit operator $\mathcal{L}^{*}$, the operator $\mathcal{L}^{*}-\lambda_{1}$ is critical, that is, the space of positive solutions to

$$
\mathcal{L}^{*} \varphi=\lambda_{1} \varphi \quad \text { in } \mathbb{R}
$$

has dimension 1.

Actually, the definition of criticality is related to the non-existence of a Green function (see [36]) but in dimension 1 it is known from [1], [26. Appendix 1] that it is equivalent to the validity of the positive Liouville property stated in Hypothesis 2 (see also [38, Theorem 4.3.4 and Proposition 5.1.3]). We will make use of the theory of critical operators only once, in order to show that (6) holds under Hypothesis 2, see Proposition 2.6. It follows that (6) holds under Hypothesis 1, which is more restrictive than Hypothesis 2 owing to the following.

Proposition 1.7. Let $\mathcal{L}$ be a self-adjoint operator on $\mathbb{R}$ which admits a positive bounded eigenfunction $\varphi$. Then, the associated eigenvalue is the generalized principal eigenvalue $\lambda_{1}$ given by (4), and there holds:

1. $\mathcal{L}-\lambda_{1}$ is critical;

2. if in addition $\inf \varphi>0$ then $\mathcal{L}^{*}-\lambda_{1}$ is critical for any limit operator $\mathcal{L}^{*}$;

3. $\inf \varphi>0$ if and only if $\varphi$ is a.p.

The above property 1 could be obtained as a consequence of one of the following stronger results: [26, Theorem A.9] or [37, Theorem 1.7]. We present below a different, simple direct proof. The almost periodicity of the coefficients is only needed in 3 (boundedness and uniform continuity would be enough to guarantee the existence of limit operators so that 2 makes sense). We point out that in [26] the hypothesis $\varphi$ bounded is replaced by an integrability condition which holds if $\varphi$ grows at most as $\sqrt{|x|}$. However, unlike boundedness, such more general condition cannot be exploited to get informations about limit operators.

Proof. Firstly, we know from [10, Theorem 1.7] that the existence of the positive bounded eigenfunction $\varphi$ implies that the associated eigenvalue is necessarily $\lambda_{1}$.

1. Let $\psi$ be a nontrivial solution to $\mathcal{L} \psi=\lambda_{1} \psi$. We can assume without loss of generality that $\psi(0) \neq 0$. Let us normalize $\varphi, \psi$ by $\varphi(0)=\psi(0)=1$. Using equation (7) for both $\varphi$ and $\psi$ we get, for all $x \in \mathbb{R}$,

$$
\int_{0}^{x}\left(a \varphi^{\prime}\right)^{\prime} \psi=\int_{0}^{x}\left(a \psi^{\prime}\right)^{\prime} \varphi
$$

from which we deduce

$$
a(x)\left(\varphi^{\prime} \psi-\psi^{\prime} \varphi\right)(x)=a(0)\left(\varphi^{\prime}-\psi^{\prime}\right)(0),
$$

or equivalently

$$
(\psi / \varphi)^{\prime}(x)=\frac{a(0)\left(\psi^{\prime}-\varphi^{\prime}\right)(0)}{a(x) \varphi^{2}(x)} .
$$

It follows that if $\left(\psi^{\prime}-\varphi^{\prime}\right)(0)$ has a sign then the function $(\psi / \varphi)^{\prime}$ has everywhere the same sign and it is bounded away from 0 since $\varphi$ is bounded. This implies that $\psi / \varphi$ changes sign 
on $\mathbb{R}$, that is, $\psi$ changes sign. As a consequence, $\varphi$ is the unique positive eigenfunction of $\mathcal{L}$ with eigenvalue $\lambda_{1}$, up to a scalar multiple, meaning that $\mathcal{L}-\lambda_{1}$ is critical.

2. Suppose that $\inf \varphi>0$. Let $\mathcal{L}^{*}$ be a limit operator defined through a sequence of translations $\left(x_{n}\right)_{n \in \mathbb{N}}$. The functions $\varphi\left(\cdot+x_{n}\right)$ converge locally uniformly (up to subsequences) to an eigenfunction of $\mathcal{L}^{*}$ with eigenvalue $\lambda_{1}$. Moreover, because $\varphi$ is bounded and has positive infimum, the same is true for such eigenfunction. Statement 1 of the proposition eventually implies that $\mathcal{L}^{*}-\lambda_{1}$ is critical.

3. An a.p. positive eigenfunction $\varphi$ is necessarily bounded and with positive infimum. Indeed, boundedness immediately follows from almost periodicity, while, supposing that $\inf \varphi=0$, one would readily obtain the contradiction $\varphi \equiv 0$ by applying the strong maximum principle to a limit operator associated with a minimizing sequence for $\varphi$ and then using the almost periodicity of $\varphi$. Conversely, let $\varphi$ be a bounded eigenfunction with positive infimum. We use the following characterization of a.p. functions due to Bochner [14]: a function $g: \mathbb{R} \rightarrow \mathbb{R}$ is a.p. if and only if from any pair of sequences $\left(x_{n}\right)_{n \in \mathbb{N}},\left(y_{n}\right)_{n \in \mathbb{N}}$ one can extract a common subsequence $\left(x_{n}^{\prime}\right)_{n \in \mathbb{N}},\left(y_{n}^{\prime}\right)_{n \in \mathbb{N}}$ such that

$$
\forall x \in \mathbb{R}, \quad \lim _{n \rightarrow \infty} g\left(x+x_{n}^{\prime}+y_{n}^{\prime}\right)=\lim _{m \rightarrow \infty}\left(\lim _{n \rightarrow \infty} g\left(x+x_{n}^{\prime}+y_{m}^{\prime}\right)\right) .
$$

Consider two sequences $\left(x_{n}\right)_{n \in \mathbb{N}},\left(y_{n}\right)_{n \in \mathbb{N}}$ in $\mathbb{R}$. By elliptic estimates, the limits

$$
\widetilde{\varphi}(x):=\lim _{n \rightarrow \infty} \varphi\left(x+x_{n}\right), \quad \widetilde{\varphi}^{*}(x):=\lim _{n \rightarrow \infty} \tilde{\varphi}\left(x+y_{n}\right), \quad \varphi^{*}(x):=\lim _{n \rightarrow \infty} \varphi\left(x+x_{n}+y_{n}\right),
$$

exist (up to subsequences) locally uniformly in $x \in \mathbb{R}$, and, applying Bochner's characterization to the coefficients $a, c$, we deduce that both $\widetilde{\varphi}^{*}$ and $\varphi^{*}$ are eigenfunctions with eigenvalue $\lambda_{1}$ of the limit operator $\mathcal{L}^{*}$ associated with (a subsequence of) the sequence of translations $\left(x_{n}+y_{n}\right)_{n \in \mathbb{N}}$. The operator $\mathcal{L}^{*}-\lambda_{1}$ is critical by statement 2 , which means that the positive functions $\widetilde{\varphi}^{*}$ and $\varphi^{*}$ coincide up to a scalar multiple $\beta>0$. Namely

$$
\forall x \in \mathbb{R}, \quad \lim _{m \rightarrow \infty}\left(\lim _{n \rightarrow \infty} \varphi\left(x+x_{n}+y_{m}\right)\right)=\beta \lim _{n \rightarrow \infty} \varphi\left(x+x_{n}+y_{n}\right) .
$$

If we show that $\beta=1$, we would infer from Bochner's characterization that $\varphi$ is a.p. To do this, we first deduce from the fact that $\varphi^{*}, \widetilde{\varphi}$ are obtained as limits of translations of $\varphi$ and that $\widetilde{\varphi}^{*}$ is a limit of translations of $\widetilde{\varphi}$ that

$$
\inf \varphi^{*} \geq \inf \varphi, \quad \sup \varphi^{*} \leq \sup \varphi, \quad \inf \widetilde{\varphi}^{*} \geq \inf \widetilde{\varphi} \geq \inf \varphi, \quad \sup \widetilde{\varphi}^{*} \leq \sup \widetilde{\varphi} \leq \sup \varphi .
$$

Now, we apply property (8) with $\left(x_{n}\right)_{n \in \mathbb{N}}$ replaced by $\left(x_{n}+y_{n}\right)_{n \in \mathbb{N}}$ and with $\left(y_{n}\right)_{n \in \mathbb{N}}$ replaced by $\left(-x_{n}-y_{n}\right)_{n \in \mathbb{N}}$. We infer the existence of some $\beta^{*}>0$ such that (up to subsequences)

$$
\forall x \in \mathbb{R}, \quad \lim _{m \rightarrow \infty} \varphi^{*}\left(x-x_{m}-y_{m}\right)=\beta^{*} \varphi(x) .
$$

This means that $\beta^{*} \varphi$ is a limit of translations of $\varphi^{*}$ and therefore

$$
\beta^{*} \inf \varphi \geq \inf \varphi^{*} \geq \inf \varphi, \quad \beta^{*} \sup \varphi \leq \sup \varphi^{*} \leq \sup \varphi .
$$

It follows that $\beta^{*}=1$ and $\inf \varphi^{*}=\inf \varphi, \sup \varphi^{*}=\sup \varphi$. With analogous arguments, considering the pair of sequences $\left(x_{n}\right)_{n \in \mathbb{N}}$ and $\left(-x_{n}\right)_{n \in \mathbb{N}}$ we derive $\inf \widetilde{\varphi}=\inf \varphi, \sup \widetilde{\varphi}=\sup \varphi$. 
Finally, starting from the function $\widetilde{\varphi}$ (which satisfies the same type of eigenvalue problem with a.p. coefficients as $\varphi$ ) and considering the sequences $\left(y_{n}\right)_{n \in \mathbb{N}}$ and $\left(-y_{n}\right)_{n \in \mathbb{N}}$, we find that $\inf \widetilde{\varphi}^{*}=\inf \widetilde{\varphi}, \sup \widetilde{\varphi}^{*}=\sup \widetilde{\varphi}$. Summing up, we have that $\inf \widetilde{\varphi}^{*}=\inf \varphi^{*}, \sup \widetilde{\varphi}^{*}=\sup \varphi^{*}$ whence $\beta=1$.

Proposition $1.8([20])$. Let $M \geq 2$ and $\omega=\left(\omega_{1}, \ldots, \omega_{M}\right) \in \mathbb{R}^{M}$ be such that

$$
\forall n \in \mathbb{Z}^{M} \backslash\{0\}, \quad|n \cdot \omega| \geq k|n|^{-\sigma} \quad \text { for some } k, \sigma>0 .
$$

Assume that $a$ and $c$ are quasiperiodic, in the sense that there exist two functions $\hat{a}, \hat{c} \in \mathcal{C}\left(\mathbb{R}^{M}, \mathbb{R}\right)$ with periods $\mathbb{Z}^{M}$ which are 1 -periodic in all directions, and $\left(\omega_{1}, \ldots, \omega_{M}\right) \in(0, \infty)^{M}$ such that $a(x)=\hat{a}\left(\omega_{1} x, \ldots, \omega_{M} x\right)$ and $c(x)=\hat{c}\left(\omega_{1} x, \ldots, \omega_{M} x\right)$ for all $x \in \mathbb{R}$. Then there exist $r=r(\sigma)$ and $\varepsilon=\varepsilon(\sigma)$ such that if $\hat{a}, \hat{c} \in \mathcal{C}^{r}\left(\mathbb{R}^{M}, \mathbb{R}\right)$ and $\|\hat{c}\|_{\mathcal{C}^{r}}<\varepsilon$, then Hypothesis 1 is satisfied.

A typical example of a function $c$ satisfying these hypotheses is $c(x)=\varepsilon(\cos (x)+\cos (\sqrt{2} x))$, with $\varepsilon$ small enough.

\section{Properties of the linearized problem}

We investigate now the properties of the eigenfunctions of the linearized operator $\mathcal{L}$ defined by (3). For any (possibly unbounded) interval $I$ we define the generalized principal eigenvalue

$$
\lambda_{1}(\mathcal{L}, I)=\inf \left\{\lambda \in \mathbb{R}, \exists \phi \in \mathcal{C}^{2}(I), \phi>0 \text { in } I, \mathcal{L} \phi \leq \lambda \phi \text { in } I\right\} .
$$

If $I$ is bounded it coincides with the classical principal eigenvalue. In the case $I=\mathbb{R}$ the definition reduces to that of $\lambda_{1}$ in (44); we will sometimes use the notation $\lambda_{1}(\mathcal{L}, \mathbb{R})$ in order to avoid ambiguity. The following characterizations hold without assuming $a, c$ to be almost periodic, but just bounded (see [1], [10]):

$$
\begin{gathered}
\lambda_{1}(\mathcal{L}, I)=\sup _{\varphi \in H_{0}^{1}(I), \varphi \neq 0} \frac{\int_{I}\left(c(x) \varphi^{2}-a(x)\left(\varphi^{\prime}\right)^{2}\right) d x}{\int_{I} \varphi^{2} d x}, \\
\lambda_{1}(\mathcal{L}, \mathbb{R})=\lim _{R \rightarrow+\infty} \lambda_{1}(\mathcal{L},(-R, R)) .
\end{gathered}
$$

From (10) it follows that $\lambda_{1}(\mathcal{L}, I)$ is nondecreasing with respect to the inclusion of intervals $I$. If $\mathcal{L}$ has a.p. coefficients then $\lambda_{1}(\mathcal{L}, \mathbb{R})$ can also be characterized through intervals invading only $\mathbb{R}_{+}$(or $\mathbb{R}_{-}$).

Proposition 2.1. There holds

$$
\lambda_{1}(\mathcal{L},(0, R)) \nearrow \lambda_{1}(\mathcal{L}, \mathbb{R}) \geq \inf c \quad \text { as } \quad R \nearrow+\infty
$$

Proof. First, it is well-known that for all $R>0$, there exists a Dirichlet principal eigenfunction, that is, $\varphi_{R} \in \mathcal{C}^{2}([0, R])$ such that $\varphi_{R}(0)=\varphi_{R}(R)=0, \varphi_{R}>0$ in $(0, R)$ and 
$\left(a(x) \varphi_{R}^{\prime}\right)^{\prime}+c(x) \varphi_{R}=\lambda_{1}(\mathcal{L},(0, R)) \varphi_{R}$ in $(0, R)$. From (10), it follows that $R \mapsto \lambda_{1}(\mathcal{L},(0, R))$ is nondecreasing and bounded from above by $\lambda_{1}(\mathcal{L}, \mathbb{R})$. Hence, one can define

$$
\lambda:=\lim _{R \rightarrow+\infty} \lambda_{1}(\mathcal{L},(0, R)) \leq \lambda_{1}(\mathcal{L}, \mathbb{R}) .
$$

The Harnack inequality, elliptic regularity estimates and a diagonal extraction imply that there exists a sequence $\left(R_{n}\right)_{n}$ such that $R_{n} \rightarrow+\infty$ and the functions $\left(\psi_{n}\right)_{n}$ defined by $\psi_{n}(x):=\varphi_{2 R_{n}}\left(x+R_{n}\right) / \varphi_{2 R_{n}}\left(R_{n}\right)$ converge to some $\psi_{\infty}$ in $\mathcal{C}^{1}(K)$ for all compact set $K \subset \mathbb{R}$. Since $a$ and $c$ are a.p., we can assume without loss of generality that there exists $a^{*}, c^{*} \in \mathcal{C}(\mathbb{R})$ such that $a\left(x+R_{n}\right) \rightarrow a^{*}(x)$ and $c\left(x+R_{n}\right) \rightarrow c^{*}(x)$ as $n \rightarrow \infty$ uniformly in $x \in \mathbb{R}$. It follows that $\psi_{\infty}$ is a weak solution of

$$
\mathcal{L}^{*} \psi_{\infty}=\lambda \psi_{\infty} \text { in } \mathbb{R}, \quad \psi_{\infty}(0)=1, \quad \psi_{\infty} \geq 0 \text { in } \mathbb{R}
$$

where $\mathcal{L}^{*}$ is the limit operator defined by $\mathcal{L}^{*} \phi:=\left(a^{*}(x) \phi^{\prime}\right)^{\prime}+c^{*}(x) \phi$. It follows that $\psi_{\infty} \in \mathcal{C}^{2}(\mathbb{R})$ and, by the strong maximum principle, that $\psi_{\infty}>0$ in $\mathbb{R}$. Then the characterization (10) yields $\lambda_{1}\left(\mathcal{L}^{*}, \mathbb{R}\right) \leq \lambda$. But since $\lambda_{1}\left(\mathcal{L}^{*}, \mathbb{R}\right)=\lambda_{1}(\mathcal{L}, \mathbb{R})$ by [7, Lemma 5.6] because $\mathcal{L}$ is a.p., we conclude that $\lambda=\lambda_{1}(\mathcal{L}, \mathbb{R})$.

Finally, taking $\varphi(x)=\cos \left(\frac{\pi}{2 R} x\right)$ in the characterization (11) we deduce that, as $R \rightarrow \infty$, $\lambda_{1}(\mathcal{L},(-R, R)) \geq \inf c+O\left(R^{-2}\right)$, whence, by (12), $\lambda_{1}(\mathcal{L}, \mathbb{R}) \geq \inf c$.

In the sequel we will make frequent use of the following technical lemma, which is an immediate consequence of Lemma 2.2 in [31]. The latter was proved for $a \equiv 1$ but the reader could easily check that it holds true for an elliptic diffusion term $a$ satisfying the hypotheses of the present paper.

Lemma 2.2. For all $\gamma>\lambda_{1}(\mathcal{L}, \mathbb{R}), x_{0} \in \mathbb{R}$ and $\varepsilon>0$, there exists $C>0$ such that any generalized subsolution $\phi \in \mathcal{C}\left(\left[x_{0},+\infty\right)\right)$ of

$$
\mathcal{L} \phi=\gamma \phi \quad \text { in }\left(x_{0},+\infty\right), \quad \lim _{x \rightarrow+\infty} \phi(x)=0,
$$

satisfies

$$
\phi(x) \leq C\left(\max \left\{\phi\left(x_{0}\right), 0\right\}\right) e^{-\left(\sqrt{\gamma-\lambda_{1}(\mathcal{L}, \mathbb{R})}-\varepsilon\right) x} .
$$

Proof of Proposition 1.3(i). The conclusion holds even when $a$ and $c$ are not a.p., but just bounded so that $\lambda_{1}(\mathcal{L}, \mathbb{R})$ is finite. The proof is very close to that of Theorem 1.1 in [31]. Take $\gamma>\lambda_{1}(\mathcal{L}, \mathbb{R})$. Hence, $\gamma>\lambda_{1}(\mathcal{L},(0, R))$ for all $R>0$, which implies that the principal eigenvalue of the operator $\mathcal{L}-\gamma$ with Dirichlet boundary conditions in $(0, R)$ is negative. There exists then a unique positive solution $\phi_{\gamma}^{R}$ of

$$
\left(a(x)\left(\phi_{\gamma}^{R}\right)^{\prime}\right)^{\prime}+(c(x)-\gamma) \phi_{\gamma}^{R}=0 \quad \text { in }(0, R), \quad \phi_{\gamma}^{R}(0)=1, \quad \phi_{\gamma}^{R}(R)=0 .
$$

By the comparison principle, the family $\left(\phi_{\gamma}^{R}\right)_{R>0}$ is increasing in $R$. By Lemma 2.2, for all $\varepsilon>0$, there exists a constant $C=C(\varepsilon, \gamma)$ so that

$$
\forall R>0, x \in(0, R), \quad \phi_{\gamma}^{R}(x) \leq C e^{-\left(\sqrt{\gamma-\lambda_{1}(\mathcal{L}, \mathbb{R})}-\varepsilon\right) x} .
$$


Hence, one can define $\phi(x):=\lim _{R \rightarrow+\infty} \phi_{\gamma}^{R}(x)$ for $x \geq 0$. This limit belongs to $\mathcal{C}^{2}([0, \infty))$ and satisfies $\left(a(x) \phi^{\prime}\right)^{\prime}+c(x) \phi=\gamma \phi$ over $(0, \infty)$ and $\phi(0)=1$. Moreover, taking $0<\varepsilon<\sqrt{\gamma-\lambda_{1}(\mathcal{L}, \mathbb{R})}$, one gets from (14) that $\lim _{x \rightarrow+\infty} \phi(x)=0$.

Next, we consider the unique $\mathcal{C}^{2}$ extension of $\phi$ to the full real line satisfying $\mathcal{L} \phi=\gamma \phi$. It is only left to check that this extension, that we still denote $\phi$, is positive. Assume that it is not true and define $x_{0}:=\sup \{x \in \mathbb{R}, \phi(x)=0\}$. Define

$$
\varphi(x):=\left\{\begin{array}{ccc}
0 & \text { if } & x<x_{0} \\
\phi(x) & \text { if } \quad x \geq x_{0}
\end{array}\right.
$$

Then $\varphi \in H_{0}^{1}(\mathbb{R})$ since $\phi$ converges exponentially to 0 as $x \rightarrow+\infty$ and $\phi^{\prime} \in L^{2}\left(x_{0}, \infty\right)$ using equation $\left(a(x) \phi^{\prime}\right)^{\prime}+c(x) \phi=\gamma \phi$. Taking $\varphi$ as a test-function in (11) yields $\lambda_{1}(\mathcal{L}, \mathbb{R}) \geq \gamma$, which is a contradiction.

Lastly, the uniqueness follows either from the characterization of the validity of the maximum principle in terms of the sign of $\lambda_{1}(\mathcal{L}, \mathbb{R})$ derived in [10, Theorems 1.6 and 1.9], or from Lemma 2.2. Indeed, for instance, applying the latter with $x_{0}=0$ and $\phi$ equal to the difference of two solutions $\phi_{1}, \phi_{2}$ of (5) , yields $\phi_{1} \leq \phi_{2}$ on $\mathbb{R}_{+}$. Then, exchanging $\phi_{1}$ and $\phi_{2}$ we eventually derive $\phi_{1} \equiv \phi_{2}$ on $\mathbb{R}_{+}$, and thus on the whole $\mathbb{R}$ by uniqueness of the Cauchy problem.

Lemma 2.3. For all $\gamma>\lambda_{1}(\mathcal{L}, \mathbb{R})$, the function $\phi_{\gamma}$ is unbounded.

Proof. Assume that $\phi_{\gamma}$ is bounded. Define

$$
\lambda_{1}^{\prime}(\mathcal{L}, \mathbb{R}):=\sup \left\{\lambda \in \mathbb{R}, \exists \varphi \in \mathcal{C}^{2}(\mathbb{R}) \cap L^{\infty}(\mathbb{R}), \phi>0 \text { in } \mathbb{R}, \mathcal{L} \phi \geq \lambda \phi \text { in } \mathbb{R}\right\} .
$$

As $\phi_{\gamma}$ is bounded, one can take $\phi=\phi_{\gamma}$ in the above formula, which gives $\lambda_{1}^{\prime}(\mathcal{L}, \mathbb{R}) \geq \gamma$. On the other hand, it has been proved in [7] that, as $\mathcal{L}$ is self-adjoint, $\lambda_{1}^{\prime}(\mathcal{L}, \mathbb{R})=\lambda_{1}(\mathcal{L}, \mathbb{R})$. This contradicts $\gamma>\lambda_{1}(\mathcal{L}, \mathbb{R})$.

Lemma 2.4. For all $\gamma>\lambda_{1}(\mathcal{L}, \mathbb{R})$, the function $\phi_{\gamma}^{\prime} / \phi_{\gamma}$ is a.p.

Proof. Take $\gamma>\lambda_{1}(\mathcal{L}, \mathbb{R})$. Consider a sequence $\left(x_{n}\right)_{n}$ in $\mathbb{R}$. Then, up to subsequences, $\left(a\left(\cdot+x_{n}\right)\right)_{n}$ and $\left(c\left(\cdot+x_{n}\right)\right)_{n}$ converge uniformly to some $a^{*}, c^{*} \in \mathcal{C}(\mathbb{R})$. The operator $\mathcal{L}^{*}$ defined by $\mathcal{L}^{*} \phi:=\left(a^{*}(x) \phi^{\prime}\right)^{\prime}+c^{*}(x) \phi$ is a limit operator associated with $\mathcal{L}$. Hence, since $a$ and $c$ are a.p., Lemma 5.6 of [7] yields $\lambda_{1}\left(\mathcal{L}^{*}, \mathbb{R}\right)=\lambda_{1}(\mathcal{L}, \mathbb{R})<\gamma$. We can therefore apply Proposition $1.3\left(\right.$ i) to $\mathcal{L}^{*}$ and infer that there exists a positive function $\phi^{*} \in \mathcal{C}^{2}(\mathbb{R})$ such that

$$
\mathcal{L}^{*} \phi^{*}=\gamma \phi^{*} \quad \text { in } \mathbb{R}, \quad \phi^{*}(0)=1, \quad \lim _{x \rightarrow+\infty} \phi^{*}(x)=0 .
$$

We prove the lemma by showing that $\phi_{\gamma}^{\prime}\left(\cdot+x_{n}\right) / \phi_{\gamma}\left(\cdot+x_{n}\right)$ converges up to subsequences to $\left(\phi^{*}\right)^{\prime} / \phi^{*}$ uniformly in $x \in \mathbb{R}$. Assume by way of contradiction that this is not the case. There exists then a sequence $\left(y_{n}\right)_{n}$ such that, up to extraction,

$$
\lim _{n \rightarrow \infty}\left|\frac{\phi_{\gamma}^{\prime}\left(y_{n}+x_{n}\right)}{\phi_{\gamma}\left(y_{n}+x_{n}\right)}-\frac{\left(\phi^{*}\right)^{\prime}\left(y_{n}\right)}{\phi^{*}\left(y_{n}\right)}\right|>0 .
$$


One can assume, always up to extraction, that as $n \rightarrow \infty,\left(a\left(\cdot+x_{n}+y_{n}\right)\right)_{n}$ and $\left(c\left(\cdot+x_{n}+y_{n}\right)\right)_{n}$ converge to some $a^{* *}$ and $c^{* *}$ uniformly in $\mathbb{R}$. It is easy to check that $a^{*}\left(\cdot+y_{n}\right) \rightarrow a^{* *}$ and $c^{*}\left(\cdot+y_{n}\right) \rightarrow c^{* *}$ as $n \rightarrow \infty$ uniformly in $x \in \mathbb{R}$.

Next, set $\psi_{n}(x):=\phi_{\gamma}\left(x+x_{n}+y_{n}\right) / \phi_{\gamma}\left(x_{n}+y_{n}\right)$. Since $\phi_{\gamma}$ satisfies $\mathcal{L} \phi_{\gamma}=\gamma \phi_{\gamma}$, the Harnack inequality together with interior elliptic estimates imply that the sequence $\left(\psi_{n}\right)_{n}$ is bounded in $\mathcal{C}^{1, \alpha}(I)$ for any $\alpha \in(0,1)$ and any bounded interval $I$. It follows from the Ascoli theorem that $\left(\psi_{n}\right)_{n \in \mathbb{N}}$ converges (up to extraction) in $\mathcal{C}_{\text {loc }}^{1}(\mathbb{R})$ to some function $\psi_{\infty}$; expressing $\psi_{n}^{\prime \prime}$ from the equation $\mathcal{L} \psi_{n}=\gamma \psi_{n}$ we deduce that the convergence actually holds in $\mathcal{C}_{\text {loc }}^{2}(\mathbb{R})$. The function $\psi_{\infty}$ is positive and satisfies

$$
\left(a^{* *}(x) \psi_{\infty}^{\prime}\right)^{\prime}+c^{* *}(x) \psi_{\infty}=\gamma \psi_{\infty} \text { in } \mathbb{R}, \quad \psi_{\infty}(0)=1 .
$$

Furthermore, we know from Lemma 2.2 that, for given $\varepsilon>0$, there is $C>0$ such that

$$
\forall x>0, \quad \phi_{\gamma}\left(x+x_{n}+y_{n}\right) \leq C \phi_{\gamma}\left(x_{n}+y_{n}\right) e^{-\left(\sqrt{\gamma-\lambda_{1}(\mathcal{L}, \mathbb{R})}-\varepsilon\right) x},
$$

and thus $\psi_{\infty}(x) \leq C e^{-\left(\sqrt{\gamma-\lambda_{1}(\mathcal{L}, \mathbb{R})}-\varepsilon\right) x}$. This implies $\lim _{x \rightarrow+\infty} \psi_{\infty}(x)=0$.

Similarly, defining $\varphi_{n}(x):=\phi^{*}\left(x+y_{n}\right) / \phi^{*}\left(y_{n}\right)$ for all $n$ and $x \in \mathbb{R}$, one can prove that, up to extraction, the sequence $\left(\varphi_{n}\right)_{n}$ converges in $\mathcal{C}_{l o c}^{2}(\mathbb{R})$ to a solution $\varphi_{\infty}$ of (16). Moreover $\lim _{x \rightarrow+\infty} \varphi_{\infty}(x)=0$ again by Lemma 2.2. Proposition 1.3 eventually yields that $\varphi_{\infty} \equiv \psi_{\infty}$. This is impossible because $\varphi_{\infty}^{\prime}(0) \neq \psi_{\infty}^{\prime}(0)$ by (15), which provides the final contradiction.

Proof of Proposition 1.3(ii). We write

$$
\frac{1}{x} \ln \phi_{\gamma}(x)=\frac{1}{x} \int_{0}^{x} \frac{\phi_{\gamma}^{\prime}(y)}{\phi_{\gamma}(y)} d y .
$$

Since the function $\phi_{\gamma}^{\prime} / \phi_{\gamma}$ is a.p. by Lemma 2.4, it is well known that the average

$$
-\mu(\gamma):=\lim _{x \rightarrow \pm \infty} \frac{1}{x} \int_{z}^{z+x} \frac{\phi_{\gamma}^{\prime}(y)}{\phi_{\gamma}(y)} d y
$$

exists uniformly in $z \in \mathbb{R}$ (see, e.g., [11, [16]). We show in the next lemma that $\mu(\gamma) \geq \sqrt{\gamma-\lambda_{1}(\mathcal{L}, \mathbb{R})}>0$, which concludes the proof of the statement.

Lemma 2.5. The function $\gamma \mapsto \mu(\gamma)$ defined on $\left(\lambda_{1}(\mathcal{L}, \mathbb{R}),+\infty\right)$ is concave, nondecreasing and there exists $C>0$ such that, for $\gamma>\lambda_{1}(\mathcal{L}, \mathbb{R})$,

$$
\begin{gathered}
\sqrt{\gamma-\lambda_{1}(\mathcal{L}, \mathbb{R})} \leq \mu(\gamma) \leq C \sqrt{\gamma}, \\
\mu(\gamma)>\underline{\mu}:=\lim _{\gamma \searrow \lambda_{1}(\mathcal{L}, \mathbb{R})} \mu(\gamma) .
\end{gathered}
$$


Proof. Take $\lambda_{1}(\mathcal{L}, \mathbb{R})<\gamma_{1}<\gamma_{2}$ and $\theta \in(0,1)$. Call $\gamma:=(1-\theta) \gamma_{1}+\theta \gamma_{2}$ and $\psi(x):=\phi_{\gamma_{1}}^{1-\theta}(x) \phi_{\gamma_{2}}^{\theta}(x)$. A straightforward computation yields

$$
\begin{aligned}
\left(a(x) \psi^{\prime}\right)^{\prime} & =-a(x) \theta(1-\theta) \psi\left(\frac{\phi_{\gamma_{1}}^{\prime}}{\phi_{\gamma_{1}}}-\frac{\phi_{\gamma_{2}}^{\prime}}{\phi_{\gamma_{2}}}\right)^{2}+a(x)(1-\theta)\left(\frac{\phi_{\gamma_{2}}}{\phi_{\gamma_{1}}}\right)^{\theta} \phi_{\gamma_{1}}^{\prime \prime}+a(x) \theta\left(\frac{\phi_{\gamma_{1}}}{\phi_{\gamma_{2}}}\right)^{1-\theta} \phi_{\gamma_{2}}^{\prime \prime} \\
& +a^{\prime}(x)(1-\theta)\left(\frac{\phi_{\gamma_{2}}}{\phi_{\gamma_{1}}}\right)^{\theta} \phi_{\gamma_{1}}^{\prime}+a^{\prime}(x) \theta\left(\frac{\phi_{\gamma_{1}}}{\phi_{\gamma_{2}}}\right)^{1-\theta} \phi_{\gamma_{2}}^{\prime} \\
& \leq(1-\theta)\left(\frac{\phi_{\gamma_{2}}}{\phi_{\gamma_{1}}}\right)^{\theta}\left(\gamma_{1}-c(x)\right) \phi_{\gamma_{1}}+\theta\left(\frac{\phi_{\gamma_{1}}}{\phi_{\gamma_{2}}}\right)^{1-\theta}\left(\gamma_{2}-c(x)\right) \phi_{\gamma_{2}} \\
& =(\gamma-c(x)) \psi .
\end{aligned}
$$

The maximum principle, together with the boundary conditions at 0 and $+\infty$, gives $\phi_{\gamma}(x) \leq \psi(x)=\phi_{\gamma_{1}}^{1-\theta}(x) \phi_{\gamma_{2}}^{\theta}(x)$ for all $x>0$. It follows that

$$
\forall x>0, \quad-\frac{1}{x} \ln \phi_{\gamma}(x) \geq-\frac{(1-\theta)}{x} \ln \phi_{\gamma_{1}}(x)-\frac{\theta}{x} \ln \phi_{\gamma_{2}}(x),
$$

whence, letting $x$ go to $+\infty$, we eventually get $\mu(\gamma) \geq(1-\theta) \mu\left(\gamma_{1}\right)+\theta \mu\left(\gamma_{2}\right)$.

Next, $\phi_{\gamma_{2}}$ is a subsolution of the equation satisfied by $\phi_{\gamma_{1}}$ in $[0, \infty)$ and thus, applying Lemma 2.2 to $\phi_{\gamma_{2}}-\phi_{\gamma_{1}}$ shows that $\phi_{\gamma_{2}} \leq \phi_{\gamma_{1}}$ in $[0, \infty)$. Hence, we obtain the monotonicity of $\gamma \mapsto \mu(\gamma)$.

Lemma 2.2 yields that, for any $\varepsilon>0$,

$$
\mu(\gamma)=-\lim _{x \rightarrow+\infty} \frac{1}{x} \ln \phi_{\gamma}(x) \geq-\lim _{x \rightarrow+\infty} \frac{C}{x}+\sqrt{\gamma-\lambda_{1}(\mathcal{L}, \mathbb{R})}-\varepsilon=\sqrt{\gamma-\lambda_{1}(\mathcal{L}, \mathbb{R})}-\varepsilon .
$$

Finally, assume by way of contradiction that there exists $\gamma>\lambda_{1}(\mathcal{L}, \mathbb{R})$ such that $\mu(\gamma)=\underline{\mu}$. Then $\mu(\gamma)=\underline{\mu}$ for all $\gamma>\lambda_{1}(\mathcal{L}, \mathbb{R})$ by monotonicity and concavity, which contradict $\mu(\gamma) \geq \sqrt{\gamma-\lambda_{1}(\mathcal{L}, \mathbb{R})}$

Lastly, it is easy to check that Lemma 2.6 of [31] holds true in our framework, even if $a$ is heterogeneous, providing a constant $C$ such that $\mu(\gamma) \leq C \sqrt{\gamma}$.

We conclude this section by the analysis of the limit of the exponent $\mu(\gamma)$ as $\gamma \searrow \lambda_{1}$.

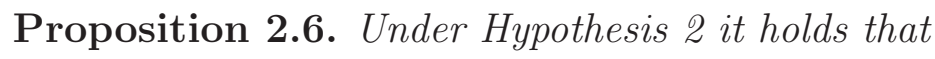

$$
\underline{\mu}:=\lim _{\gamma \searrow \lambda_{1}} \mu(\gamma)=0
$$

Proof. Consider the analogue $\widetilde{\phi}_{\gamma}$ of $\phi_{\gamma}$ but with imposed decay at $-\infty$, namely,

$$
\mathcal{L} \widetilde{\phi}_{\gamma}=\gamma \widetilde{\phi}_{\gamma} \text { in } \mathbb{R}, \quad \widetilde{\phi}_{\gamma}(0)=1, \quad \lim _{x \rightarrow-\infty} \widetilde{\phi}_{\gamma}(x)=0
$$

The function $\widetilde{\phi}_{\gamma}(-x)$ fulfils the same properties as $\phi_{\gamma}$. In particular the limit

$$
\widetilde{\mu}(\gamma):=\lim _{x \rightarrow \pm \infty} \frac{1}{x} \ln \widetilde{\phi}_{\gamma}(x)
$$


exists and it is positive. Consider the combination $\varphi_{\gamma}:=\sqrt{\widetilde{\phi}_{\gamma} \phi_{\gamma}}$. The same computation as in the proof of Lemma 2.5 reveals that $\varphi_{\gamma}$ satisfies the equation 1

$$
(\mathcal{L}-\gamma) \varphi_{\gamma}=-\frac{1}{4} a(x) q_{\gamma}^{2} \varphi_{\gamma}, \quad \text { with } q_{\gamma}=\frac{\widetilde{\phi}_{\gamma}^{\prime}}{\widetilde{\phi}_{\gamma}}-\frac{\phi_{\gamma}^{\prime}}{\phi_{\gamma}} .
$$

We claim that $q_{\gamma}$ converges uniformly to 0 in $\mathbb{R}$ as $\gamma \searrow \lambda_{1}$. Suppose by contradiction that this is not the case. Then there exist $\varepsilon>0$ and two sequences $\left(\gamma_{n}\right)_{n}$ and $\left(x_{n}\right)_{n}$ such that $\gamma_{n} \searrow \lambda_{1}$ and $\left|q_{\gamma_{n}}\left(x_{n}\right)\right| \geq \varepsilon$ for all $n \in \mathbb{N}$. Applying the Harnack inequality and then elliptic estimates to $\widetilde{\phi}_{\gamma_{n}}$ and $\phi_{\gamma_{n}}$ we see that the $\left(q_{\gamma_{n}}\right)_{n}$ are equibounded and equicontinuous on $\mathbb{R}$. Passing to the limit along (a subsequence of) the translations by $x_{n}$ in (19), we find that the functions $\varphi_{\gamma}\left(\cdot+x_{n}\right) / \varphi_{\gamma}\left(x_{n}\right)$ converge to a positive solution $\varphi^{*}$ of

$$
\left(\mathcal{L}^{*}-\lambda_{1}\right) \varphi^{*}=-\frac{1}{4} a^{*}(x) q^{2} \varphi^{*}
$$

where $\mathcal{L}^{*}$ is a limit operator associated with $\mathcal{L}$ and $a^{*}, q$ are the limits of $\left(a\left(\cdot+x_{n}\right)\right)_{n}$ and $\left(q_{\gamma_{n}}\left(\cdot+x_{n}\right)\right)_{n}$ respectively. Since $|q(0)| \geq \varepsilon$, we deduce that the operator $\mathcal{L}^{*}-\lambda_{1}$ admits a positive supersolution which is not a solution. This contradicts the criticality of $\mathcal{L}^{*}-\lambda_{1}$ due to [38, Theorem 3.9 at p. 152]. We have therefore shown that $q_{\gamma} \rightarrow 0$ uniformly in $\mathbb{R}$ as $\gamma \searrow \lambda_{1}$. Finally, because

$$
\widetilde{\mu}(\gamma)+\mu(\gamma)=\lim _{x \rightarrow+\infty} \frac{1}{x} \int_{0}^{x} q_{\gamma} \leq\left\|q_{\gamma}\right\|_{\infty}
$$

and $\widetilde{\mu}(\gamma), \mu(\gamma)$ are positive, we infer that both $\widetilde{\mu}(\gamma)$ and $\mu(\gamma)$ tend to 0 as $\gamma \searrow \lambda_{1}$.

Remark 1. As Hypothesis 1 implies Hypothesis 2 by Proposition 1.7, it follows from Proposition 2.6 that $\mu=0$ when Hypothesis 1 is fulfilled. Owing to Lemma 2.5, this implies in particular that $\bar{w}^{*}$ is attained. Therefore, Theorem 1.4 is a consequence of Theorem 1.5.

Remark 2. As explained in Section 1.3. Hypotheses 1 and 2 are fulfilled if $c$ is constant. However, in such case, the limit of $\mu(\gamma)$ can be derived directly without using the theory of critical operators. Assume indeed that $c$ is a positive constant. On one hand, taking $\phi \equiv 1$ in (10) yields $\lambda_{1} \leq c$, on the other, we know from Proposition 2.1 that $\lambda_{1} \geq c$. Thus, $\lambda_{1}=c$. We use now the same type of argument as Zlatos in the proof of [50, Theorem 1.1], even if our aim is different. Let $\delta>0$, take $\gamma>c$ and define $u(x):=a(x) \phi_{\gamma}^{\prime}(x) / \phi_{\gamma}(x)$. This function is a.p. - being the product of two a.p. functions, see, e.g., [11, Theorem 1.13] - and satisfies

$$
u^{\prime}+u^{2} / a(x)=\gamma-c \quad \text { in } \mathbb{R} .
$$

Moreover, as $\left(a(x) \phi_{\gamma}^{\prime}\right)^{\prime}=(\gamma-c) \phi_{\gamma}>0$ in $\mathbb{R}$, the function $\phi_{\gamma}$ does not admit any local maximum and, as $\phi_{\gamma}(+\infty)=0$, it is thus nonincreasing. Hence $u$ is nonpositive. Now, it is easy to check that

$$
u^{2}(x) \leq(\gamma-c) \sup _{\mathbb{R}} a \quad \text { for all } x \in \mathbb{R}
$$

\footnotetext{
${ }^{1}$ The function $\frac{1}{4} a(x) q_{\gamma}^{2}$ is an optimal Hardy weight in the sense of [15, which implies in particular that the operator $\mathcal{L}-\gamma+\frac{1}{4} a(x) q_{\gamma}^{2}$ is critical. However, one cannot deduce from this the criticality of $\mathcal{L}-\lambda_{1}$ (and of its limit operators), but we need to impose it as an assumption.
} 
because if the above inequality failed for some $x_{0} \in \mathbb{R}, u$ would be decreasing in $\left(-\infty, x_{0}\right)$, contradicting the almost periodicity. It follows that

$$
\left|\phi_{\gamma}^{\prime}(x) / \phi_{\gamma}(x)\right| \leq \frac{\sqrt{\gamma-c} \sup _{\mathbb{R}} \sqrt{a}}{\inf _{\mathbb{R}} a} \quad \text { for all } x \in \mathbb{R}
$$

Recalling that $\mu(\gamma)$ is equal to the average of $\phi_{\gamma}^{\prime} / \phi_{\gamma}$, we derive

$$
|\mu(\gamma)| \leq \frac{\sqrt{\gamma-c} \sup _{\mathbb{R}} \sqrt{a}}{\inf _{\mathbb{R}} a} \quad \text { for all } \gamma>c
$$

from which (6) follows because $\lambda_{1}=c$.

\section{Construction of the fronts}

\subsection{A preliminary result on a.p. linear operators}

Consider an arbitrary elliptic operator defined for all $\phi \in \mathcal{C}^{2}(\mathbb{R})$ by

$$
\mathcal{M} \phi:=a(x) \phi^{\prime \prime}+b(x) \phi^{\prime}+c(x) \phi
$$

with $a, b, c$ a.p. in $x$ and $\inf _{\mathbb{R}} a>0$.

Lemma 3.1. Assume that there exist $\delta^{\prime} \in \mathbb{R}$ and a positive function $\zeta \in \mathcal{C}^{2}(\mathbb{R})$ so that

$$
-\mathcal{M} \zeta \geq \delta^{\prime} \zeta \quad \text { in } \mathbb{R}, \quad \lim _{|x| \rightarrow+\infty} \frac{1}{x} \ln \zeta(x)=0 .
$$

Then for all $\delta<\delta^{\prime}$, there exists $\theta \in \mathcal{C}^{2}(\mathbb{R}) \cap L^{\infty}(\mathbb{R})$ satisfying

$$
-\mathcal{M} \theta \geq \delta \theta \quad \text { in } \mathbb{R}, \quad \inf _{\mathbb{R}} \theta>0
$$

Proof. Our proof makes use of the following two generalized principal eigenvalues introduced in [8]:

$$
\begin{aligned}
& \underline{\lambda_{1}}:=\sup \left\{\lambda \mid \exists \zeta \in \mathcal{C}^{2}(\mathbb{R}), \zeta>0, \lim _{|x| \rightarrow+\infty} \frac{1}{x} \ln \zeta(x)=0 \text { such that } \mathcal{M} \zeta \geq \lambda \zeta \text { in } \mathbb{R}\right\}, \\
& \overline{\lambda_{1}}:=\inf \left\{\lambda \mid \exists \zeta \in \mathcal{C}^{2}(\mathbb{R}), \zeta>0, \lim _{|x| \rightarrow+\infty} \frac{1}{x} \ln \zeta(x)=0 \text { such that } \mathcal{M} \zeta \leq \lambda \zeta \text { in } \mathbb{R}\right\} .
\end{aligned}
$$

It has been proved in [8] that $\underline{\lambda_{1}} \leq \overline{\lambda_{1}}$. The hypothesis of the lemma reads $\overline{\lambda_{1}} \leq-\delta^{\prime}$.

We will now use the same type of arguments as in [8] in order to show that $\lambda_{1}=\overline{\lambda_{1}}$, from which we will construct the desired supersolution $\theta$. As the result of this lemma plays a central role in the proof of our main result, we briefly sketch the argument here for the sake of completeness. Consider the family of equations defined, for $\varepsilon>0$, by:

$$
a(x) u_{\varepsilon}^{\prime \prime}+a(x)\left(u_{\varepsilon}^{\prime}\right)^{2}+b(x) u_{\varepsilon}^{\prime}+c(x)=\varepsilon u_{\varepsilon} \quad \text { in } \mathbb{R} .
$$


For all $\varepsilon>0$, as $c$ is uniformly bounded, there exists some large $M_{\varepsilon}$ such that $-M_{\varepsilon}$ is a subsolution and $M_{\varepsilon}$ is a supersolution of (23). It follows from Perron's method that there exists a (unique) bounded solution $u_{\varepsilon} \in \mathcal{C}^{2}(\mathbb{R})$ of equation (23). It has been proved by Lions and Souganidis in [22, Lemma 3.3] that $\lambda=\lim _{\varepsilon \rightarrow 0} \varepsilon u_{\varepsilon}(x)$ exists uniformly in $x \in \mathbb{R}$.

The function $\zeta:=e^{u_{\varepsilon}}$ satisfies $\mathcal{M} \zeta=\varepsilon u_{\varepsilon} \zeta$. Taking it as a test function in the definitions of $\underline{\lambda_{1}}$ and $\overline{\lambda_{1}}$ and letting $\varepsilon$ go to 0 yields $\underline{\lambda_{1}} \geq \lambda \geq \overline{\lambda_{1}}$ and thus $\underline{\lambda_{1}}=\lambda=\overline{\lambda_{1}}$. In particular, $\lambda \leq-\delta^{\prime}$. Take $\delta<\delta^{\prime}$ and choose $\varepsilon>0$ small enough so that $\left|\overline{\varepsilon u}_{\varepsilon}(x)-\lambda\right|<\delta^{\prime}-\delta$ for all $x \in \mathbb{R}$. Then, the function $\theta:=\zeta=e^{u_{\varepsilon}}$ satisfies

$$
\mathcal{M} \theta=\varepsilon u_{\varepsilon} \theta \leq\left(\lambda+\delta^{\prime}-\delta\right) \theta \leq-\delta \theta \quad \text { in } \mathbb{R} .
$$

As $u_{\varepsilon}$ is bounded, $\theta$ is also bounded and satisfies $\inf _{\mathbb{R}} \theta>0$.

\subsection{Construction of sub and supersolutions}

In the sequel, for $\gamma>\lambda_{1}$, we will let $\phi_{\gamma}$ denote the function given by Proposition 1.3. We further set

$$
\sigma_{\gamma}:=-\frac{\phi_{\gamma}^{\prime}}{\phi_{\gamma}} .
$$

Lemma 3.2. Assume that $w^{*}, \underline{w}$ defined in Theorem 1.5 satisfy $w^{*}<\underline{w}$. Then $w^{*}$ is $a$ minimum. Moreover, for all $w \in\left(w^{*}, \underline{w}\right)$, there exists $\gamma>\lambda_{1}$ such that $w=\gamma / \mu(\gamma)$ and $w>\gamma^{\prime} / \mu\left(\gamma^{\prime}\right)$ for $\gamma^{\prime}-\gamma>0$ small enough.

Proof. Recall that $\lambda_{1} \geq \inf c>0$ by Proposition 2.1. On one hand, by hypothesis,

$$
\lim _{\gamma \rightarrow \lambda_{1}} \frac{\gamma}{\mu(\gamma)}=\frac{\lambda_{1}}{\underline{\mu}}=\underline{w}>w^{*}=\inf _{\gamma>\lambda_{1}} \frac{\gamma}{\mu(\gamma)} .
$$

On the other, the upper bound for $\mu$ in (18) yields $\gamma / \mu(\gamma) \rightarrow+\infty$ as $\gamma \rightarrow+\infty$. Hence, the function $\gamma \mapsto \gamma / \mu(\gamma)$, which is continuous because $\gamma \mapsto \mu(\gamma)$ is concave by Lemma 2.5, admits a minimum on $\left(\lambda_{1},+\infty\right)$. Let $\gamma^{*}$ be a minimizing point.

Take $w \in\left(w^{*}, \underline{w}\right)$ and define $f(\gamma):=w \mu(\gamma)-\gamma$ for $\gamma \in\left(\lambda_{1},+\infty\right)$. There holds

$$
\lim _{\gamma \rightarrow+\infty} f(\gamma)=-\infty, \quad \lim _{\gamma \searrow \lambda_{1}} f(\gamma)=w \underline{\mu}-\lambda_{1}<0 .
$$

Moreover, $f$ is concave and $f\left(\gamma^{*}\right)>0$. It follows that $f$ is strictly increasing for $\gamma$ less than its first maximal point $\gamma_{M}$, and that $f\left(\gamma_{M}\right)>0$. The unique zero of $f$ in $\left(\lambda_{1}, \gamma_{M}\right)$ provides us with the desired $\gamma$.

Throughout this section, we take $w \in\left(w^{*}, \underline{w}\right)$ and we let $\gamma>\lambda_{1}$ be as in Lemma $\underline{3.2}$. For a given a.p. function $\sigma \in \mathcal{C}^{1}(\mathbb{R})$, we define the operator

$$
\mathcal{L}_{\sigma} \phi:=e^{\int_{0}^{x} \sigma} \mathcal{L}\left(e^{-\int_{0}^{x} \sigma} \phi\right)=\left(a(x) \phi^{\prime}\right)^{\prime}-2 a(x) \sigma \phi^{\prime}+\left(a(x) \sigma^{2}-(a(x) \sigma)^{\prime}+c(x)\right) \phi .
$$

Proposition 3.3. There exist $\delta>0, \varepsilon \in(0,1)$ and a function $\theta \in \mathcal{C}^{2}(\mathbb{R}) \cap L^{\infty}(\mathbb{R})$ satisfying

$$
\inf _{\mathbb{R}} \theta>0, \quad-\mathcal{L}_{(1+\varepsilon) \sigma_{\gamma}} \theta \geq(\delta-(1+\varepsilon) \gamma) \theta \quad \text { in } \mathbb{R}
$$


Proof. Due to our choice of $\gamma$, there exists $\varepsilon \in(0,1)$ so that

$$
\frac{\gamma}{\mu(\gamma)}>\frac{(1+\varepsilon) \gamma}{\mu((1+\varepsilon) \gamma)}
$$

Define

$$
F(\kappa):=\frac{1}{\mu(\gamma)}-\frac{1+\varepsilon}{\mu(\gamma+\kappa)} .
$$

Then $F(\gamma \varepsilon)=1 / \mu(\gamma)-(1+\varepsilon) / \mu((1+\varepsilon) \gamma)>0$ and $F(0)=-\varepsilon / \mu(\gamma)<0$. As $\mu$ is concave, it is continuous and thus $F$ is continuous. Hence, there exists $\kappa \in(0, \varepsilon \gamma)$ so that $F(\kappa)=0$.

Consider now the function

$$
\zeta(x):=\frac{\phi_{\gamma+\kappa}(x)}{\phi_{\gamma}^{1+\varepsilon}(x)} \text { for all } x \in \mathbb{R} .
$$

Keeping in mind that $e^{-(1+\varepsilon) \int_{0}^{x} \sigma_{\gamma}}=\phi_{\gamma}^{1+\varepsilon}$, we find that the positive function $\zeta$ satisfies, in $\mathbb{R}$,

$$
\mathcal{L}_{(1+\varepsilon) \sigma_{\gamma}} \zeta=e^{(1+\varepsilon) \int_{0}^{x} \sigma_{\gamma}} \mathcal{L}\left(e^{-(1+\varepsilon) \int_{0}^{x} \sigma_{\gamma}} \zeta\right)=\frac{1}{\phi_{\gamma}^{1+\varepsilon}} \mathcal{L}\left(\zeta \phi_{\gamma}^{1+\varepsilon}\right)=\frac{1}{\phi_{\gamma}^{1+\varepsilon}} \mathcal{L}\left(\phi_{\gamma+\kappa}\right)=(\gamma+\kappa) \zeta
$$

It follows that

$$
-\left(\mathcal{L}_{(1+\varepsilon) \sigma_{\gamma}}-(1+\varepsilon) \gamma\right) \zeta=(\varepsilon \gamma-\kappa) \zeta \quad \text { in } \mathbb{R}
$$

Moreover,

$$
-\frac{1}{x} \ln \zeta(x)=-\frac{1}{x} \ln \phi_{\gamma+\kappa}(x)+\frac{1+\varepsilon}{x} \ln \phi_{\gamma}(x),
$$

and we know from Proposition 1.3(ii) that, as $x \rightarrow+\infty$, the right-hand side tends to $\mu(\gamma+\kappa)-(1+\varepsilon) \mu(\gamma)$, which is equal to 0 by the definition of $\kappa$. Notice that we obtain the same limit when $x \rightarrow-\infty$ thanks to the fact that $\mu(\gamma)$ is the uniform average of the a.p. function $-\phi_{\gamma}^{\prime} / \phi_{\gamma}$, that is, (17) holds uniformly with respect to $z \in \mathbb{R}$, and likewise $\mu(\gamma+\kappa)$ is the uniform average of $-\phi_{\gamma+\kappa}^{\prime} / \phi_{\gamma+\kappa}$. Hence, the hypotheses of Lemma 3.1 are fulfilled by the operator $\mathcal{M}=\mathcal{L}_{(1+\varepsilon) \sigma_{\gamma}}-(1+\varepsilon) \gamma$ with $\delta^{\prime}=\varepsilon \gamma-\kappa>0$, and the statement of the proposition follows, up to showing that $\mathcal{M}$ has a.p. coefficients. Since $a$ and $\sigma_{\gamma}$ are a.p. by hypothesis and Lemma 2.4, the only nontrivial check concerns the term $\left(a \sigma_{\gamma}\right)^{\prime}$. We know from [16, Theorem 1.16] that, in order to prove that $\left(a \sigma_{\gamma}\right)^{\prime}$ is a.p., it is sufficient to show that $a \sigma_{\gamma}$ is uniformly continuous, which is readily achieved applying the Harnack inequality and a priori estimates to the function $\phi_{\gamma}$.

Define for all $(t, x) \in \mathbb{R} \times \mathbb{R}$ :

$$
\begin{gathered}
\bar{u}(t, x):=\min \left\{1, \phi_{\gamma}(x) e^{\gamma t}\right\}, \\
\underline{u}(t, x):=\max \left\{0, \phi_{\gamma}(x) e^{\gamma t}-A \theta(x) \phi_{\gamma}^{1+\varepsilon}(x) e^{(1+\varepsilon) \gamma t}\right\},
\end{gathered}
$$

where $\varepsilon$ and $\theta$ are given by Proposition 3.3 and $A$ is a positive constant that will be chosen later. 
Proposition 3.4. There exists a (weak) solution $u \in \mathcal{C}(\mathbb{R} \times \mathbb{R})$ of (1) satisfying $\underline{u} \leq u \leq \bar{u}$ in $\mathbb{R} \times \mathbb{R}$. Moreover, $u=u(t, x)$ is increasing in $t$.

Proof. Direct computation reveals that $\phi_{\gamma}(x) e^{\gamma t}$ is a supersolution on the whole $\mathbb{R} \times \mathbb{R}$ of (1), hence $\bar{u}$ is a generalized supersolution of the same equation. Take $(t, x) \in \mathbb{R} \times \mathbb{R}$ so that $\underline{u}(t, x)>0$ and set for short $\zeta:=\phi_{\gamma}(x) e^{\gamma t}$. One has:

$$
\begin{aligned}
\underline{u}_{t}-\left(a(x) \underline{u}_{x}\right)_{x}-c(x) \underline{u} & =-A(1+\varepsilon) \gamma \theta \phi_{\gamma}^{1+\varepsilon} e^{(1+\varepsilon) \gamma t}+A e^{(1+\varepsilon) \gamma t} \phi_{\gamma}^{1+\varepsilon} \mathcal{L}_{(1+\varepsilon) \sigma_{\gamma}} \theta \\
& =A \zeta^{1+\varepsilon}\left[\mathcal{L}_{(1+\varepsilon) \sigma_{\gamma}} \theta-(1+\varepsilon) \gamma \theta\right] \\
& \leq-A \delta \theta \zeta^{1+\varepsilon} .
\end{aligned}
$$

Therefore, as 0 obviously solves (11), for $\underline{u}$ to be a subsolution it is sufficient to choose $A$ so large that, for all $(t, x)$ such that $\underline{u}(t, x)>0$, one has

$$
A \delta \theta \zeta^{1+\varepsilon} \geq c \zeta^{2} .
$$

Observe that $\underline{u}(t, x)>0$ if and only if $A \theta(t, x) \zeta^{\varepsilon}(t, x)<1$, i.e., $\zeta^{\varepsilon-1}(t, x)>(A \theta(t, x))^{1 / \varepsilon-1}$, whence the goal is achieved for

$$
A \geq \frac{\sup _{\mathbb{R}} c^{\varepsilon}}{\delta^{\varepsilon} \inf _{\mathbb{R}} \theta} .
$$

The above observation also shows that $\underline{u}<(A \theta)^{-1 / \varepsilon}$, and thus $A$ can be chosen in such a way that, in addition, $\underline{u}<1$, whence $\underline{u} \leq \bar{u}$. A standard argument then provides us with a solution $\underline{u} \leq u \leq \bar{u}$. Let us recall such argument and show that $u$ inherits from $\bar{u}$ the monotonicity in $t$.

Define the sequence of function $\left(u_{n}\right)_{n}$ in the following way: $u_{n}$ is the solution to (1) for $t>-n$ with initial condition $u_{n}(-n, x)=\bar{u}(-n, x)$. By the comparison principle, the $u_{n}$ satisfy

$$
\forall t>-n, x \in \mathbb{R}, \quad \underline{u}(t, x) \leq u_{n}(t, x) \leq \bar{u}(t, x) .
$$

Thus, for $m, n \in \mathbb{N}$ with $m<n$ and for any $0<h<1$, using the monotonicity of $\bar{u}$ we get

$$
u_{m}(-m, x)=\bar{u}(-m, x) \geq \bar{u}(-m-h, x) \geq u_{n}(-m-h, x) .
$$

Observe that $u_{n}(\cdot-h, \cdot)$ is also a solution of (1), whence the comparison principle yields

$$
\forall m<n, 0<h<1, t>-m, x \in \mathbb{R}, \quad u_{m}(t, x) \geq u_{n}(t-h, x) .
$$

By interior parabolic estimates, $\left(u_{n}\right)_{n}$ converges locally uniformly (up to subsequences) to an entire solution $\underline{u} \leq u \leq \bar{u}$ of (11). Then, passing to the limit as $m, n \rightarrow \infty$ (along a subsequence) in (24) we eventually infer that $u(t, x) \geq u(t-h, x)$ for all $(t, x) \in \mathbb{R} \times \mathbb{R}$ and $0<h<1$. This means that $u(t, x)$ is nondecreasing in $t$. If the monotonicity were not strict, the parabolic strong maximum principle would imply that $u$ is constant in time, contradicting the fact that $\underline{u} \leq u \leq \bar{u}$. 


\subsection{Asymptotics of the profile $U$}

We define

$$
U(z, x):=u\left(\frac{1}{\gamma} \int_{0}^{x} \sigma_{\gamma}-z, x\right) \text { for all }(z, x) \in \mathbb{R} \times \mathbb{R},
$$

where $u$ is the function given by Proposition 3.4 and, we recall, $\sigma_{\gamma}:=-\phi_{\gamma}^{\prime} / \phi_{\gamma}$.

Lemma 3.5. The function $U=U(z, x)$ is decreasing in $z$ and satisfies

$$
\lim _{z \rightarrow+\infty} U(z, x)=0, \quad \lim _{z \rightarrow-\infty} U(z, x)=1, \quad \text { uniformly in } x \in \mathbb{R} .
$$

Proof. The monotonicity in $z$ of $U$ follows from the monotonicity in $t$ of $u$. One has

$$
U(z, x) \leq \bar{u}\left(\frac{1}{\gamma} \int_{0}^{x} \sigma_{\gamma}-z, x\right) \leq \phi_{\gamma}(x) \exp \left(\int_{0}^{x} \sigma_{\gamma}-\gamma z\right)=e^{-\gamma z} .
$$

Similarly, for $(z, x) \in \mathbb{R} \times \mathbb{R}$,

$$
\begin{aligned}
U(z, x) & \geq \underline{u}\left(\frac{1}{\gamma} \int_{0}^{x} \sigma_{\gamma}-z, x\right) \geq e^{-\gamma z}-A \theta(x) \phi_{\gamma}^{1+\varepsilon}(x) e^{(1+\varepsilon) \int_{0}^{x} \sigma_{\gamma}-(1+\varepsilon) \gamma z} \\
& \geq e^{-\gamma z}-A\left(\sup _{\mathbb{R}} \theta\right) e^{-(1+\varepsilon) \gamma z} .
\end{aligned}
$$

Namely, $U$ satisfies

$$
\forall(z, x) \in \mathbb{R} \times \mathbb{R}, \quad e^{-\gamma z}\left(1-M e^{-\varepsilon \gamma z}\right) \leq U(z, x) \leq e^{-\gamma z},
$$

for some positive constant $M$. From the second inequality we deduce that $U(z, x) \rightarrow 0$ as $z \rightarrow+\infty$ uniformly with respect to $x \in \mathbb{R}$. From the first one, we see that $\inf _{x \in \mathbb{R}} U(z, x)>0$ for $z$ large enough, and therefore, because of the monotonicity in $z$,

$$
\forall z \in \mathbb{R}, \quad \inf _{(-\infty, z] \times \mathbb{R}} U>0 .
$$

In particular, the following quantity is positive:

$$
\vartheta:=\lim _{z \rightarrow-\infty}\left(\inf _{x \in \mathbb{R}} U(z, x)\right) .
$$

To conclude the proof we need to show that $\vartheta=1$. Let $\left(x_{n}\right)_{n}$ in $\mathbb{R}$ be such that $U\left(-n, x_{n}\right) \rightarrow \vartheta$ as $n \rightarrow \infty$. Consider the family of functions $\left(p^{n}\right)_{n}$ defined by

$$
p^{n}(t, x):=u\left(\frac{1}{\gamma} \int_{0}^{x_{n}} \sigma_{\gamma}+n-t, x_{n}+x\right)=U\left(\frac{1}{\gamma} \int_{x_{n}}^{x_{n}+x} \sigma_{\gamma}-n+t, x_{n}+x\right) .
$$

These functions satisfy $p^{n}(0,0)=U\left(-n, x_{n}\right) \rightarrow \vartheta$ as $n \rightarrow \infty$ and, for $(t, x) \in \mathbb{R} \times \mathbb{R}$, $\liminf _{n \rightarrow \infty} p^{n}(t, x) \geq \vartheta$. Moreover, by interior parabolic estimates, they converge, up to subsequences, to a function $p^{\infty}$ satisfying $\partial_{t} p^{\infty}-\left(a^{*}(x) p_{x}^{\infty}\right)_{x}=c^{*}(x) p^{\infty}\left(1-p^{\infty}\right)$ in $\mathbb{R} \times \mathbb{R}$, where $a^{*}$ and $c^{*}$ are the uniform limits of $\left(a\left(\cdot+x_{n}\right)\right)_{n}$ and $\left(c\left(\cdot+x_{n}\right)\right)_{n}$ (up to extraction). Evaluating this equation at the minimum point $(0,0)$ of $p^{\infty}$ yields $c^{*}(0) \vartheta(1-\vartheta) \leq 0$. Since $c^{*}(0) \geq \inf _{\mathbb{R}} c>0$ and $0<\vartheta \leq 1$, we eventually get $\vartheta=1$. 


\subsection{Almost periodicity of the profile $U$}

The function $U$ defined by (25) solves

$$
-\sigma^{2}(x) a(x) \partial_{z z} U-\partial_{x}\left(a(x) \partial_{x} U\right)+2 \sigma(x) a(x) \partial_{z x} U-\left((a \sigma)^{\prime}(x)+1\right) \partial_{z} U=c(x) U(1-U),
$$

for $z \in \mathbb{R}, x \in \mathbb{R}$, with $\sigma:=\sigma_{\gamma} / \gamma$. We say that a function $V$ is a sub (resp. super) solution of (29) in a domain $\mathcal{O} \subset \mathbb{R}^{2}$ if $v(t, x):=V\left(\int_{0}^{x} \sigma-t, x\right)$ is a classical sub (resp. super) solution of (11) for $\left(\int_{0}^{x} \sigma-t, x\right) \in \mathcal{O}$.

The following result is an easy consequence of the weak Harnack inequality.

Lemma 3.6. Let $I$ be an open interval, $\sigma \in W^{1, \infty}(\mathbb{R})$ and $U^{1}, U^{2}$ be respectively a sub and a supersolution of (29) which are uniformly continuous and satisfy $0 \leq U^{1} \leq U^{2} \leq 1$ in $I \times \mathbb{R}$. If

$$
\exists z \in I, \quad \inf _{x \in \mathbb{R}}\left(U^{2}-U^{1}\right)(z, x)=0,
$$

then

$$
\forall z^{\prime} \in I, \quad z^{\prime}>z, \quad \inf _{x \in \mathbb{R}}\left(U^{2}-U^{1}\right)\left(z^{\prime}, x\right)=0 .
$$

Proof. Assume that there exists $z \in I$ such that $\inf _{x \in \mathbb{R}}\left(U^{2}-U^{1}\right)(z, x)=0$ and take $z^{\prime} \in I$, $z^{\prime}>z$. Let $\left(x_{n}\right)_{n}$ be such that $\left(U^{2}-U^{1}\right)\left(z, x_{n}\right) \rightarrow 0$ as $n \rightarrow \infty$. Take $h>0$ small enough so that $z-h, z^{\prime}+2 h \in I$ and take $0<\rho<h /\|\sigma\|_{L^{\infty}(\mathbb{R})}$. Then, for $i \in\{1,2\}$ and $n \in \mathbb{N}$, the function

$$
u_{n}^{i}(t, x):=U^{i}\left(\int_{0}^{x} \sigma\left(x_{n}+s\right) d s-t, x_{n}+x\right)
$$

is well defined in $\left[-z^{\prime}-h,-z\right] \times(-\rho, \rho)$. We define there the function $w_{n}:=u_{n}^{2}-u_{n}^{1}$, which is nonnegative and satisfies

$$
\partial_{t} w_{n}-\partial_{x}\left(a\left(x+x_{n}\right) \partial_{x} w_{n}\right) \geq c\left(x_{n}+x\right)\left(1-u_{n}^{2}-u_{n}^{1}\right) w_{n}, \quad-z^{\prime}-h \leq t \leq-z,|x|<\rho .
$$

Therefore, taking $z<z_{1}<z_{2}<z^{\prime}$, the parabolic weak Harnack inequality (see e.g. Theorem 7.37 in [21]) provides two constants $p, C>0$ such that

$$
\forall n \in \mathbb{N}, \quad\left\|w_{n}\right\|_{L^{p}\left(\left(-z^{\prime},-z_{2}\right) \times\left(-\frac{\rho}{2}, \frac{\rho}{2}\right)\right)} \leq C_{\left(-z_{1},-z\right) \times\left(-\frac{\rho}{2}, \frac{\rho}{2}\right)} w_{n} \leq C w_{n}(-z, 0) .
$$

Whence, since $\lim _{n \rightarrow \infty} w_{n}(-z, 0)=0$, the $\left(w_{n}\right)_{n}$ converge to 0 in $L^{p}\left(\left(-z^{\prime},-z_{2}\right) \times\left(-\frac{\rho}{2}, \frac{\rho}{2}\right)\right)$. One readily deduces from the equicontinuity of the $\left(w_{n}\right)_{n}$ that the above $L^{p}$ convergence to 0 can hold only if $w_{n}\left(-z^{\prime}, 0\right) \rightarrow 0$ as $n \rightarrow \infty$. We eventually infer that $\inf _{x \in \mathbb{R}}\left(U^{2}-U^{1}\right)\left(z^{\prime}, x\right)=0$.

The proof of the almost periodicity of $U$ is based on a sliding method.

Proposition 3.7. The profile $U=U(z, x)$ is a.p. in $x$ uniformly with respect to $z \in \mathbb{R}$. 
Proof. Consider a sequence $\left(x_{n}\right)_{n}$ in $\mathbb{R}$. By the almost periodicity of $a, c$ and $\sigma$, we can assume that, up to extraction of a subsequence, $\left(a\left(\cdot+x_{n}\right)\right)_{n},\left(c\left(\cdot+x_{n}\right)\right)_{n}$ and $\left(\sigma\left(\cdot+x_{n}\right)\right)_{n}$ converge uniformly in $\mathbb{R}$. We claim that $\left(U\left(\cdot, \cdot+x_{n}\right)\right)_{n}$ converges uniformly in $\mathbb{R} \times \mathbb{R}$. Assume by contradiction that it is not the case. There exist then two subsequences $\left(x_{n}^{1}\right)_{n},\left(x_{n}^{2}\right)_{n}$ of $\left(x_{n}\right)_{n}$ and some sequences $\left(y_{n}\right)_{n},\left(z_{n}\right)_{n}$ in $\mathbb{R}$ such that

$$
\liminf _{n \rightarrow \infty}\left(U\left(z_{n}, x_{n}^{1}+y_{n}\right)-U\left(z_{n}, x_{n}^{2}+y_{n}\right)\right)>0 .
$$

By (26),$\left(z_{n}\right)_{n}$ is bounded. Then, letting $\zeta$ be one of its limit points, the uniform continuity of $U$ yields

$$
\liminf _{n \rightarrow \infty}\left(U\left(\zeta, x_{n}^{1}+y_{n}\right)-U\left(\zeta, x_{n}^{2}+y_{n}\right)\right)>0 .
$$

For $i=1,2$ and $n \in \mathbb{N}$, define

$$
u_{n}^{i}(t, x):=U\left(\int_{0}^{x} \sigma\left(x_{n}^{i}+y_{n}+s\right) d s-t, x_{n}^{i}+y_{n}+x\right) .
$$

We see that the $u_{n}^{i}$ satisfy (11) with $a$ and $c$ translated by $x_{n}^{i}+y_{n}+x$, and that

$$
\liminf _{n \rightarrow \infty}\left(u_{n}^{1}-u_{n}^{2}\right)(-\zeta, 0)>0 .
$$

Moreover, $\left(a\left(\cdot+x_{n}^{1}+y_{n}\right)\right)_{n},\left(c\left(\cdot+x_{n}^{1}+y_{n}\right)\right)_{n}$ and $\left(\sigma\left(\cdot+x_{n}^{1}+y_{n}\right)\right)_{n}$ converge (up to subsequences) to some functions $a^{*}, c^{*}$ and $\sigma^{*}$ uniformly in $\mathbb{R}$. Since $\left(a\left(\cdot+x_{n}\right)\right)_{n},\left(c\left(\cdot+x_{n}\right)\right)_{n}$ and $\left(\sigma\left(\cdot+x_{n}\right)\right)_{n}$ converge uniformly in $\mathbb{R}$, it turns out that also $\left(a\left(\cdot+x_{n}^{2}+y_{n}\right)\right)_{n},\left(c\left(\cdot+x_{n}^{2}+y_{n}\right)\right)_{n}$ and $\left(\sigma\left(\cdot+x_{n}^{2}+y_{n}\right)\right)_{n}$ converge uniformly to $a^{*}, c^{*}$ and $\sigma^{*}$. By parabolic estimates, we find that the $\left(u_{n}^{i}\right)_{n}$ converge (up to subsequences) locally uniformly to some functions $v^{i}$ which satisfy (11) with $a$ and $c$ replaced by $a^{*}$ and $c^{*}$. We also have that $\left(v^{1}-v^{2}\right)(-\zeta, 0)>0$. It is straightforward to check that the functions $V^{i}$ defined by $V^{i}(z, x):=v^{i}\left(\int_{0}^{x} \sigma^{*}-z, x\right)$ satisfy

$$
\forall z, x \in \mathbb{R}, \quad V^{i}(z, x)=\lim _{n \rightarrow \infty} U\left(z, x_{n}^{i}+y_{n}+x\right),
$$

and that they solve (29) with $a, c$ and $\sigma$ replaced by $a^{*}, c^{*}$ and $\sigma^{*}$ respectively. Set

$$
\kappa^{*}:=\sup _{\mathbb{R} \times \mathbb{R}} \frac{V^{1}}{V^{2}} .
$$

The fact that both $V^{1}$ and $V^{2}$ inherit from $U$ the uniform convergence to 1 as $z \rightarrow-\infty$, as well as the inequalities (27), imply that $V^{1} / V^{2} \rightarrow 1$ as $z \rightarrow \pm \infty$ uniformly in $x \in \mathbb{R}$. Since they also inherit (28), we infer that $\kappa^{*}$ is finite. On the other hand, we know that $\kappa^{*}>1$ because $\left(V^{1}-V^{2}\right)(\zeta, 0)=\left(v^{1}-v^{2}\right)(-\zeta, 0)>0$. As a consequence, using the uniform continuity of $V^{1}$ and $V^{2}$, we find that $\kappa^{*}$ is attained at some finite $\bar{z}$, in the sense that

$$
\sup _{x \in \mathbb{R}} \frac{V^{1}}{V^{2}}(\bar{z}, x)=\sup _{\mathbb{R} \times \mathbb{R}} \frac{V^{1}}{V^{2}}=\kappa^{*} .
$$

Direct computation shows that $\kappa^{*} V^{2}$ is a supersolution of (29), because $\kappa^{*}>1$. We can therefore apply Lemma 3.6, deducing that

$$
\forall z^{\prime}>z, \quad \inf _{x \in \mathbb{R}}\left(\kappa^{*} V^{2}-V^{1}\right)\left(z^{\prime}, x\right)=0 .
$$

From this, taking $z^{\prime}$ sufficiently large, we get a contradiction with (27). 


\subsection{Existence of fronts with supercritical speeds}

We can now complete the proof of Theorem 1.5)(i) in the case $w>w^{*}$.

Proposition 3.8. For any $w \in\left(w^{*}, \underline{w}\right)$, the solution

$$
u(t, x)=U\left(\frac{1}{\gamma} \int_{0}^{x} \sigma_{\gamma}(y) d y-t, x\right)
$$

constructed before, with $\gamma$ given by Lemma 3.2, is a generalized transition front with average speed $\gamma /\left\langle\sigma_{\gamma}\right\rangle=w$.

Proof. Recall that, because $\sigma_{\gamma}$ is a.p., the following limit exists uniformly in $a \in \mathbb{R}$ :

$$
\left\langle\sigma_{\gamma}\right\rangle=\lim _{x \rightarrow \pm \infty} \frac{1}{x} \int_{a}^{a+x} \sigma_{\gamma} .
$$

By construction, c.f. in particular (17) and Lemma 3.2, we have that

$$
\left\langle\sigma_{\gamma}\right\rangle=\left\langle-\phi_{\gamma}^{\prime} / \phi_{\gamma}\right\rangle=\mu(\gamma)=\gamma / w
$$

Since $\gamma / w>0$, this allows us to define $X(t)$, for $t \in \mathbb{R}$, as the smallest value for which $\int_{0}^{X(t)} \sigma_{\gamma}=\gamma t$. One has

$$
u(t, x+X(t))=U\left(\frac{1}{\gamma} \int_{X(t)}^{X(t)+x} \sigma_{\gamma}(y) d y, x+X(t)\right),
$$

whence, using (30) and (26), we eventually derive (2).

Lastly, one has $\int_{X(s)}^{X(t)} \sigma_{\gamma}=\gamma(t-s)$. Hence, $\gamma|t-s| \leq\left\|\sigma_{\gamma}\right\|_{\infty}|X(t)-X(s)|$ for all $s, t \in \mathbb{R}$, and therefore $|X(t)-X(s)| \rightarrow+\infty$ as $t-s \rightarrow+\infty$. It follows that

$$
\frac{\gamma(t-s)}{X(t)-X(s)}=\frac{1}{X(t)-X(s)} \int_{X(s)}^{X(t)} \sigma_{\gamma} \rightarrow\left\langle\sigma_{\gamma}\right\rangle \quad \text { as } t-s \rightarrow+\infty
$$

that is, $u$ has average speed $\gamma /\left\langle\sigma_{\gamma}\right\rangle=w$.

\subsection{Non-existence of fronts with speed less than $w^{*}$}

The following proposition immediately implies statements (ii) of Theorems 1.4 and 1.5 .

Proposition 3.9. Let $u$ be a generalized transition front of equation (1) and let $X$ be such that (2) holds. Then

$$
\forall s \in \mathbb{R}, \quad \liminf _{t \rightarrow+\infty} \frac{X(s+t)-X(s)}{t} \geq w^{*} .
$$

In particular, there exists no generalized transition front with average speed $w<w^{*}$. 
Proof. For any $p \in \mathbb{R}$, we consider the second order elliptic operator with a.p. coefficients

$$
\mathcal{L}_{p} \phi:=e^{p x} \mathcal{L}\left(e^{-p x} \phi\right)
$$

and we let $\underline{\lambda_{1}}\left(\mathcal{L}_{p}\right)$ and $\overline{\lambda_{1}}\left(\mathcal{L}_{p}\right)$ denote the generalized principal eigenvalues defined by (21) and (22) with $\mathcal{M}=\mathcal{L}_{p}$. We know from the proof of Lemma 3.1 that $\lambda_{1}\left(\mathcal{L}_{p}\right)=\overline{\lambda_{1}}\left(\mathcal{L}_{p}\right)$, and we call this quantity $k_{p}$ in order to shorten the notation. It has been proved by Berestycki and the first author in [8, Theorem 2.3] that if $v$ is a solution of equation (1) associated with a continuous initial datum with compact support $v_{0} \geq 0, \not \equiv 0$, then $\lim _{t \rightarrow+\infty} v(t, w t)=1$ for all $w \in\left(0, \min _{p>0} k_{p} / p\right)$.

Consider now a generalized transition front $u$. Then there exists $\varepsilon_{0}>0$ such that

$$
\forall s \in \mathbb{R}, \quad 2 \varepsilon_{0}<u(s, X(s))<1-2 \varepsilon_{0},
$$

see (1.10) in [5, Theorem 1.2]. Since $u$ is uniformly continuous by regularity estimates, there is $\ell>0$ such that $u(s, X(s)+x)>\varepsilon_{0}$ for all $s \in \mathbb{R}$ and $|x|<\ell$. Take $s \in \mathbb{R}$. Comparing with the solution $v$ of equation (1) for $t>s$, associated with a datum at time $s \in \mathbb{R}$ supported in $(X(s)-\ell, X(s)+\ell)$ and smaller than $\varepsilon_{0}$, we get that $u(s+t, x) \geq v(s+t, x)$ for all $t>0$, $x \in \mathbb{R}$, and thus

$$
\forall w \in\left(0, \min _{p>0} k_{p} / p\right), \quad \lim _{t \rightarrow+\infty} u(s+t, X(s)+w t)=1 .
$$

The definition of generalized transition fronts then yields that there exists $L>0$ such that, for $t$ large enough (depending on $s$ ), $X(s)+w t \leq X(s+t)+L$. This gives

$$
\liminf _{t \rightarrow+\infty} \frac{X(s+t)-X(s)}{t} \geq w .
$$

As this holds for all $w \in\left(0, \min _{p>0} k_{p} / p\right)$, we get the conclusion providing we could show that $\min _{p>0} k_{p} / p=w^{*}$, where, we recall, $w^{*}:=\inf _{\gamma>\lambda_{1}} \gamma / \mu(\gamma)$ with $\mu(\gamma)$ given by Proposition 1.3,

It has also been proved in [8] that $k_{p} \geq \lambda_{1}$ (Lemma 5.1), $k_{0}=\lambda_{1}$ (Step 2 of the proof of Theorem 5.1) and that $\mu:\left(\lambda_{1},+\infty\right) \rightarrow(\mu,+\infty)$ is an homeomorphism with inverse $p \mapsto k_{p}$ (see the proof of [8, Theorem 5.1]). Hence, we could rewrite

$$
w^{*}=\inf _{\gamma>\lambda_{1}(\mathcal{L}, \mathbb{R})} \frac{\gamma}{\mu(\gamma)}=\inf _{p>\underline{\mu}} \frac{k_{p}}{p} .
$$

This concludes the proof if $\underline{\mu}=0$. Otherwise, since $k_{p} \geq \lambda_{1}, k_{0}=k_{\underline{\mu}}=\lambda_{1}$ and $p \mapsto k_{p}$ is convex, one has $k_{p}=\lambda_{1}$ for all $p \in(0, \underline{\mu})$. In particular, $k_{p} / p \geq \lambda_{1} / \underline{\mu}$ for $p \in(0, \underline{\mu})$. We thus conclude that

$$
w^{*}=\inf _{p>\underline{\mu}} \frac{k_{p}}{p}=\inf _{p>0} \frac{k_{p}}{p} .
$$




\subsection{The critical front}

Remark 3. We can always assume that the function $X$ for which a given front satisfies (2) is uniformly Lipschitz-continuous (and actually even $\mathcal{C}^{\infty}$ ). Indeed, we know from [18, Proposition 4.2] that

$$
\sup _{(t, s) \in \mathbb{R}^{2},|t-s| \leq 1}|X(t)-X(s)|<+\infty .
$$

Then, defining $\hat{X}$ as the linear interpolation of the function $X$ restricted to $\mathbb{Z}$, one readily sees that $\hat{X} \in W^{1, \infty}(\mathbb{R})$ and that (2) holds true with $X$ replaced by $\hat{X}$, because, denoting the integer part of $t \in \mathbb{R}$ by $[t]$, there holds

$$
\sup _{t \in \mathbb{R}}|X(t)-\hat{X}(t)| \leq \sup _{t \in \mathbb{R}}|X(t)-X([t])|+\sup _{t \in \mathbb{R}}|\hat{X}(t)-\hat{X}([t])|<+\infty .
$$

Of course, one could use a smooth interpolation in order to get $\hat{X} \in \mathcal{C}^{\infty}(\mathbb{R})$.

Proofs of Theorems 1.4 and 1.5 part (i) when $w=w^{*}$. We now assume that $w^{*}<\underline{w}$ and consider the critical travelling wave $u$ associated with equation (11), in the sense introduced by the first author in [28], normalized by $u(0,0)=1 / 2$. Theorem 3.6 of [28] yields that $u$ is a generalized transition front (called spatial travelling wave in [28]), which is increasing with respect to $t$ (by Proposition 3.5 of [28]). It follows in particular that (2) holds with $X: \mathbb{R} \rightarrow \mathbb{R}$ such that $u(t, X(t))=1 / 2$ for all $t \in \mathbb{R}$. Moreover, the proof of this earlier result yields that if another generalized transition front satisfies (2) with a function $Y$ instead of $X$, then there exists $L>0$ such that, for all $s_{0} \in \mathbb{R}$, one can find $s_{1} \in \mathbb{R}$ so that

$$
\forall t>0, \quad X\left(s_{0}+t\right)-X\left(s_{0}\right) \leq Y\left(s_{1}+t\right)-Y\left(s_{1}\right)+L .
$$

Considering any generalized transition front with supercritical average speed $w \in\left(w^{*}, \underline{w}\right)$ constructed before, we thus obtain

$$
\limsup _{t \rightarrow+\infty} \sup _{s_{0} \in \mathbb{R}} \frac{X\left(s_{0}+t\right)-X\left(s_{0}\right)}{t} \leq \lim _{t \rightarrow+\infty} \sup _{s_{1} \in \mathbb{R}} \frac{Y\left(s_{1}+t\right)-Y\left(s_{1}\right)+L}{t}=w .
$$

This being true for $w$ arbitrarily close to $w^{*}$, we get

$$
\limsup _{t \rightarrow+\infty} \sup _{s \in \mathbb{R}} \frac{X(s+t)-X(s)}{t} \leq w^{*} .
$$

By Remark 3, we know that there is a uniformly Lipschitz-continuous function, that we still call $X$, for which $u$ fulfils (2). Since this function is obtained as a bounded perturbation of the previous $X$, (31) holds true (we just lose the information $u(t, X(t))=1 / 2$ ). This allows us to rewrite (31) in terms of the upper mean of $X^{\prime} \in L^{\infty}(\mathbb{R})$, which is defined by

$$
\left\lceil X^{\prime}\right\rceil:=\lim _{t \rightarrow+\infty} \sup _{s \in \mathbb{R}} \int_{s}^{s+t} X^{\prime}(\tau) d \tau \text {. }
$$

Namely, we have $\left\lceil X^{\prime}\right\rceil \leq w^{*}$. The notion of upper mean, together with that of least mean:

$$
\left\lfloor X^{\prime}\right\rfloor:=\lim _{t \rightarrow+\infty} \inf _{s \in \mathbb{R}} \int_{s}^{s+t} X^{\prime}(\tau) d \tau
$$


has been introduced in [29]. Actually, the above formulation for the least mean - from which the analogous one for the upper mean immediately follows - is not the original definition of [29], but comes from Proposition 3.1 there, which shows in particular the existence of the limit. Clearly, $u$ has an average speed $w$ if and only if $\left\lfloor X^{\prime}\right\rfloor=\left\lceil X^{\prime}\right\rceil=w$. Thus, in order to conclude the proof, we need to show that $\left\lfloor X^{\prime}\right\rfloor \geq w^{*}$.

To achieve our goal, we make use of another characterization of the least mean, provided by [30, Proposition 4.4], which involves the $\omega$-limit set of the function. It implies the existence of a sequence $\left(s_{n}\right)_{n}$ such that $X^{\prime}\left(\cdot+s_{n}\right)$ converges weakly- $\star$ in $L^{\infty}(\mathbb{R})$ to a function $g$ satisfying

$$
\lim _{t \rightarrow+\infty} \frac{1}{t} \int_{0}^{t} g(\tau) d \tau=\left\lfloor X^{\prime}\right\rfloor .
$$

Define the sequence of functions $\left(u_{n}\right)_{n}$ by

$$
u_{n}(t, x):=u\left(t+s_{n}, x+X\left(s_{n}\right)\right) .
$$

It follows from interior parabolic estimates that $\left(u_{n}\right)_{n}$ converges locally uniformly to a function $\widetilde{u}$ satisfying (2) with $X(t)=\int_{0}^{t} g$. Moreover, $a\left(\cdot+X\left(s_{n}\right)\right), a^{\prime}\left(\cdot+X\left(s_{n}\right)\right), c\left(\cdot+X\left(s_{n}\right)\right)$ converge uniformly in $\mathbb{R}$ (up to subsequences) to some functions $\widetilde{a}, b=(\widetilde{a})^{\prime}, \widetilde{c}$ and therefore $\widetilde{u}$ is a generalized transition front of the equation

$$
v_{t}-\left(\widetilde{a}(x) v_{x}\right)_{x}=\widetilde{c}(x) v(1-v), \quad t \in \mathbb{R}, x \in \mathbb{R} .
$$

This equation fulfils the same set of standing assumptions as (11). In particular, Proposition 1.3 holds true for the operator $\phi \mapsto \widetilde{\mathcal{L}} \phi:=\left(\widetilde{a}(x) \phi^{\prime}\right)^{\prime}+\widetilde{c}(x) \phi$ and, for all $\gamma>\lambda_{1}(\widetilde{\mathcal{L}}, \mathbb{R})$, provides us with a unique positive solution to

$$
\widetilde{\mathcal{L}} \widetilde{\phi}_{\gamma}=\gamma \widetilde{\phi}_{\gamma} \text { in } \mathbb{R}, \quad \widetilde{\phi}_{\gamma}(0)=1, \quad \lim _{x \rightarrow+\infty} \widetilde{\phi}_{\gamma}(x)=0
$$

which satisfies in addition $\widetilde{\mu}(\gamma):=-\lim _{x \rightarrow+\infty} \frac{1}{x} \ln \widetilde{\phi}_{\gamma}(x)>0$.

Let us show that $\lambda_{1}(\widetilde{\mathcal{L}}, \mathbb{R})=\lambda_{1}(\mathcal{L}, \mathbb{R})$ and that $\widetilde{\mu} \equiv \mu$. First, the operator $\widetilde{\mathcal{L}}$ is a limit operator associated with $\mathcal{L}$ in the sense of [7], and therefore Lemma 5.6 there yields $\lambda_{1}(\widetilde{\mathcal{L}}, \mathbb{R})=\lambda_{1}(\mathcal{L}, \mathbb{R})$. Next, consider the solution $\phi_{\gamma}$ provided by Proposition 1.3. The sequence $\phi_{\gamma}\left(\cdot+X\left(s_{n}\right)\right) / \phi_{\gamma}\left(X\left(s_{n}\right)\right)$ converges in $\mathcal{C}_{\text {loc }}^{2}(\mathbb{R})$ to a nonnegative function $\psi$ satisfying $\widetilde{\mathcal{L}} \psi=\gamma \psi$ in $\mathbb{R}$ and $\psi(0)=1$. It follows from the strong maximum principle that $\psi$ is positive. We then compute

$$
\lim _{x \rightarrow+\infty} \frac{1}{x} \ln \psi(x)=\lim _{x \rightarrow+\infty} \frac{1}{x} \int_{0}^{x} \frac{\psi^{\prime}}{\psi}=\lim _{x \rightarrow+\infty} \lim _{n \rightarrow \infty} \frac{1}{x} \int_{X\left(s_{n}\right)}^{X\left(s_{n}\right)+x} \frac{\phi_{\gamma}^{\prime}}{\phi_{\gamma}}=-\mu(\gamma),
$$

where we have used that the a.p. function $\phi_{\gamma}^{\prime} / \phi_{\gamma}$ satisfies (17) uniformly in $z \in \mathbb{R}$. Since $\mu(\gamma)>0$, this shows that $\psi$ decays to 0 at $+\infty$ and therefore, by uniqueness, it coincides with $\widetilde{\phi}_{\gamma}$. We eventually infer that $\widetilde{\mu}(\gamma)=\mu(\gamma)$. We can then apply Proposition 3.9 to the front $\widetilde{u}$ and obtain

$$
\inf _{\gamma>\lambda_{1}(\widetilde{\mathcal{L}}, \mathbb{R})} \frac{\gamma}{\widetilde{\mu}(\gamma)}=w^{*} \leq \liminf _{t \rightarrow+\infty} \frac{\int_{0}^{t} g(\tau) d \tau}{t}=\left\lfloor X^{\prime}\right\rfloor .
$$

This concludes the proof. 


\subsection{Proof of Lemma 1.6.}

Proof of Lemma 1.6. For all $c_{0}>-\inf c$, let $\mu_{c_{0}}(\gamma)$ be the decay rate provided by Proposition 1.3(ii) but associated with $c(x)+c_{0}$ instead of $c(x)$. It is straightforward to see that $\lambda_{1}\left(\mathcal{L}+c_{0}, \mathbb{R}\right)=\lambda_{1}(\mathcal{L}, \mathbb{R})+c_{0}$ and that $\phi_{\gamma}$ satisfies

$$
\left(a(x) \phi_{\gamma}^{\prime}\right)^{\prime}+\left(c(x)+c_{0}\right) \phi_{\gamma}=\left(\gamma+c_{0}\right) \phi_{\gamma} \quad \text { in } \mathbb{R} .
$$

Hence, it immediately follows that $\mu_{c_{0}}\left(\gamma+c_{0}\right)=\mu(\gamma)$ and then that $\underline{\mu}$ does not change when one adds $c_{0}$ to $c(x)$.

Now take $\gamma>\lambda_{1}(\mathcal{L}, \mathbb{R})$. As $\mu(\gamma)>\underline{\mu}$ by Lemma 2.5, for $c_{0}$ large enough we have that

$$
\mu(\gamma)\left(\lambda_{1}(\mathcal{L}, \mathbb{R})+c_{0}\right)>\underline{\mu}\left(\gamma+c_{0}\right) .
$$

In other words, setting $\widetilde{\gamma}:=\gamma+c_{0}$, one gets

$$
\frac{\mu_{c_{0}}(\widetilde{\gamma})}{\widetilde{\gamma}}>\frac{\underline{\mu}}{\lambda_{1}\left(\mathcal{L}+c_{0}, \mathbb{R}\right)}
$$

from which the result follows by taking the supremum over $\widetilde{\gamma}>\lambda_{1}\left(\mathcal{L}+c_{0}, \mathbb{R}\right)$.

\section{Compliance with Ethical Standards}

The authors declare that there are no conflicts of interest.

\section{References}

[1] S. Agmon. On positivity and decay of solutions of second order elliptic equations on Riemannian manifolds. in Methods of functional analysis and theory of elliptic equations (Naples, 1982), pp. 19-52, Liguori, Naples, 1983.

[2] D.G. Aronson, and H.F. Weinberger. Multidimensional nonlinear diffusions arising in population genetics. Adv. Math., 30:33-76, 1978.

[3] H. Berestycki, and F. Hamel. Front propagation in periodic excitable media. Comm. Pure Appl. Math., 55:949-1032, 2002.

[4] H. Berestycki, and F. Hamel. Generalized traveling waves for reaction-diffusion equations. Perspectives in Nonlinear Partial Differential Equations. In honor of H. Brezis, Contemp. Math. 446, Amer. Math. Soc., pages 101-123, 2007.

[5] H. Berestycki, and F. Hamel. Generalized transition waves and their properties. Communications on Pure and Applied Mathematics, 65(5):592-648, 2012.

[6] H. Berestycki, F. Hamel, and L. Roques. Analysis of the periodically fragmented environment model : II - biological invasions and pulsating traveling fronts. J. Math. Pures Appl., 84:1101-1146, 2005. 
[7] H. Berestycki, F. Hamel, and L. Rossi. Liouville-type results for semilinear elliptic equations in unbounded domains. Ann. Mat. Pura Appl., 186 (4):469-507, 2007.

[8] H. Berestycki, and G. Nadin. Spreading speeds for one-dimensional monostable reactiondiffusion equations. J. Math. Phys., 53(11), 2012.

[9] H. Berestycki, L. Nirenberg, and S. R. S. Varadhan. The principal eigenvalue and maximum principle for second-order elliptic operators in general domains. Comm. Pure Appl. Math., 47(1):47-92, 1994.

[10] H. Berestycki, and L. Rossi. Generalizations and properties of the principal eigenvalue of elliptic operators in unbounded domain. Comm. Pure Appl. Math., 68(6):1014-1065, 2015.

[11] A. S. Besicovitch. Almost periodic functions. Dover Publications, Inc., New York, 1955.

[12] J.-M. Bony. Principe du maximum dans les espaces de Sobolev. C. R. Acad. Sci. Paris Sér. A-B, 265:A333-A336, 1967.

[13] S. Bochner. Beitrage zur theorie der fastperiodischen funktionen. Math. Ann., 96:119147, 1926.

[14] S. Bochner. A new approach to almost periodicity. Proc. Nat. Acad. Sci. U.S.A., 48:2039-2043, 1962.

[15] B. Devyver, M. Fraas, and Y. Pinchover. Optimal Hardy weight for second-order elliptic operator: an answer to a problem of Agmon. J. Funct. Anal., 266(7):4422-4489, 2014.

[16] A. M. Fink. Almost periodic differential equations. Springer-Verlag, Berlin, 1974. Lecture Notes in Mathematics, Vol. 377.

[17] R. A. Fisher. The advance of advantageous genes. Ann. Eugenics, 7:335-369, 1937.

[18] F. Hamel, and L. Rossi. Transition fronts for the Fisher-KPP equation. To appear on Trans. Amer. Math. Soc.

[19] A.N. Kolmogorov, I.G. Petrovsky, and N.S. Piskunov. Etude de l'équation de la diffusion avec croissance de la quantité de matière et son application à un problème biologique. Bulletin Université d'Etat à Moscou (Bjul. Moskowskogo Gos. Univ.), 1-26, 1937.

[20] S. M. Kozlov. Ground states of quasiperiodic operators. Dokl. Akad. Nauk SSSR, 271(3):532-536, 1983.

[21] G. M. Lieberman. Second order parabolic differential equations. World Scientific Publishing Co. Inc., River Edge, NJ, 1996.

[22] P.-L. Lions and P. E. Souganidis. Homogenization of degenerate second-order PDE in periodic and almost periodic environments and applications. Ann. Inst. H. Poincaré Anal. Non Linéaire, 22(5):667-677, 2005. 
[23] B. Lou, and X. Chen. Traveling waves of a curvature flow in almost periodic media. J. Differential Equations, 247(8):2189-2208, 2009.

[24] A. Mellet, J. Nolen, J.-M. Roquejoffre, and L. Ryzhik. Stability of generalized transitions fronts. Comm. Partial Differential Equations, 34:521-552, 2009.

[25] A. Mellet, J.-M. Roquejoffre, and Y. Sire. Generalized fronts for one-dimensionnal reaction-diffusion equations. Discrete Contin. Dyn. Syst., 26(1):303-312, 2009.

[26] M. Murata. Structure of positive solutions to $(-\Delta+V) u=0$ in $\mathbf{R}^{n}$. Duke Math. J., 53(4):869-943, 1986.

[27] G. Nadin. Traveling fronts in space-time periodic media. J. Math. Pures Appl., 92:232$262,2009$.

[28] G. Nadin. Critical travelling waves for general heterogeneous reaction-diffusion equations. Ann. Inst. H. Poincaré Anal. Non Linéaire, in press, 2014.

[29] G. Nadin, and L. Rossi. Propagation phenomena for time heterogeneous KPP reactiondiffusion equations. J. Math. Pures Appl. (9), 98(6):633-653, 2012.

[30] G. Nadin and L. Rossi. Transition waves for Fisher-KPP equations with general timeheterogeneous and space-periodic coefficients. Anal. PDE, 8(6):1351-1377, 2015.

[31] J. Nolen. A central limit theorem for pulled fronts in a random medium. Net. Heter. Media, 6(2):167-194, 2011.

[32] J. Nolen, J.-M. Roquejoffre, L. Ryzhik, and A. Zlatoš. Existence and non-existence of Fisher-KPP transition fronts. Archive for Rational Mechanics and Analysis, 203(1):217$246,2012$.

[33] J. Nolen, M. Rudd, and J. Xin. Existence of KPP fronts in spatially-temporally periodic advection and variational principle for propagation speeds, Dynamics of PDE. 2(1):1-24, 2005.

[34] J. Nolen, and L. Ryzhik. Traveling waves in a one-dimensional heterogeneous medium. Ann. Inst. H. Poincaré Anal. Non Linéaire, 26(3):1021-1047, 2009.

[35] R. D. Nussbaum, and Y. Pinchover. On variational principles for the generalized principal eigenvalue of second order elliptic operators and some applications. J. Anal. Math., 59:161-177, 1992. Festschrift on the occasion of the 70th birthday of Shmuel Agmon.

[36] Y. Pinchover. On positive solutions of second-order elliptic equations, stability results, and classification. Duke Math. J., 57(3):955-980, 1988.

[37] Y. Pinchover. A Liouville-type theorem for Schrödinger operators. Comm. Math. Phys., 272(1):75-84, 2007. 
[38] R. G. Pinsky. Positive harmonic functions and diffusion, volume 45 of Cambridge Studies in Advanced Mathematics. Cambridge University Press, Cambridge, 1995.

[39] M. H. Protter, and H. F. Weinberger. On the spectrum of general second order operators. Bull. Amer. Math. Soc., 72:251-255, 1966.

[40] L. Rossi. Liouville type results for periodic and almost periodic linear operators. Ann. Institut H. Poincaré, Analyse Non Linéaire, 26:2481-2502, 2009.

[41] L. Rossi, and L. Ryzhik. Transition waves for a class of space-time dependent monostable equations. Commun. Math. Sci., 12:879-900, 2014.

[42] W. Shen. Traveling Waves in Time Almost Periodic Structures Governed by Bistable Nonlinearities, I. Stability and Uniqueness. J. Diff. Equations, 159(1):1-54, 1999.

[43] W. Shen. Travelling waves in time almost periodic structures governed by bistable nonlinearities. II. Existence. J. Diff. Equations, 159(1):55-101, 1999.

[44] W. Shen. Traveling waves in time dependent bistable equations. Differential Integral Equations, 19(3):241-278, 2006.

[45] W. Shen. Existence, uniqueness, and stability of generalized traveling waves in time dependent monostable equations. J. Dynam. Differential Equations, 23(1):1-44, 2011.

[46] W. Shen. Existence of generalized traveling waves in time recurrent and space periodic monostable equations. J. of Applied Analysis and Computation, 1(1):69-93, 2011.

[47] W. Shen. Existence of Generalized Traveling Waves in Time Recurrent and Space Periodic Monostable Equations. Journal of Applied Mathematics and Computation, 1:6994, 2011.

[48] E. Sorets, and T. Spencer. Positive Lyapunov Exponents for Schrodinger Operators with Quasi-Periodic Potentials Commun. Math. Phys., 142:543-566, 1991.

[49] J. Xin. Existence of planar flame fronts in convective-diffusive periodic media. Arch. Ration. Mech. Anal., 121:205-233, 1992.

[50] A. Zlatoš. Transition fronts in inhomogeneous Fisher-KPP reaction-diffusion equations. J. Math. Pures Appl., 98:89-102, 2012.

[51] A. Zlatoš. Generalized traveling waves in disordered media: existence, uniqueness, and stability. Arch. Ration. Mech. Anal., 208(2):447-480, 2013.

[52] A. Zlatoš. Propagation of reaction in inhomogeneous media. preprint, 2014. 